\title{
Doping liquid xenon with light elements
}

Hugh Lippincott, Fermilab

\section{COFI}

November 29, 2018 


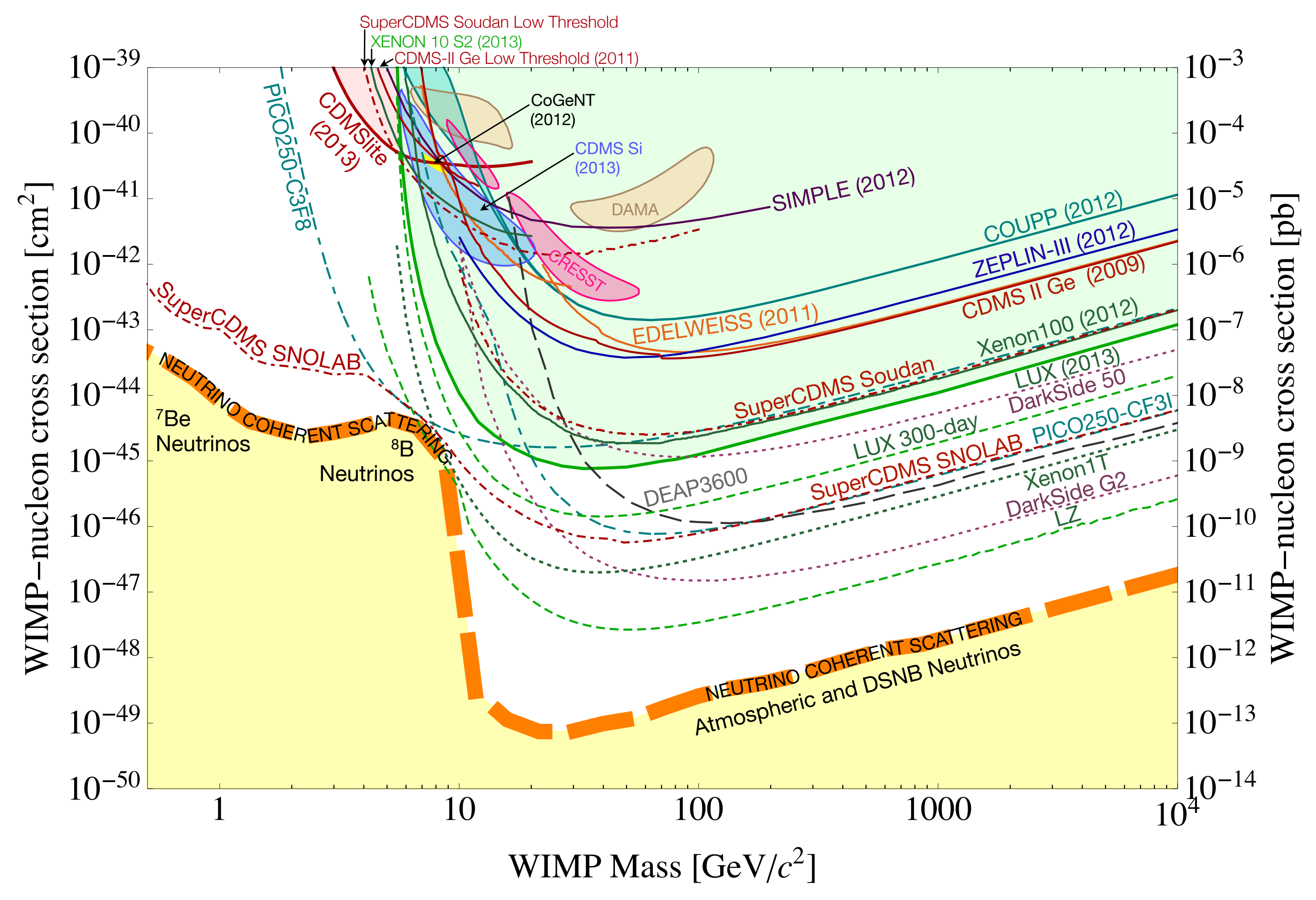

- Limited at low mass by detector threshold

- Limited at high mass by density

- Eventually limited by neutrinos 


\section{So where are we? (LZ edition)}

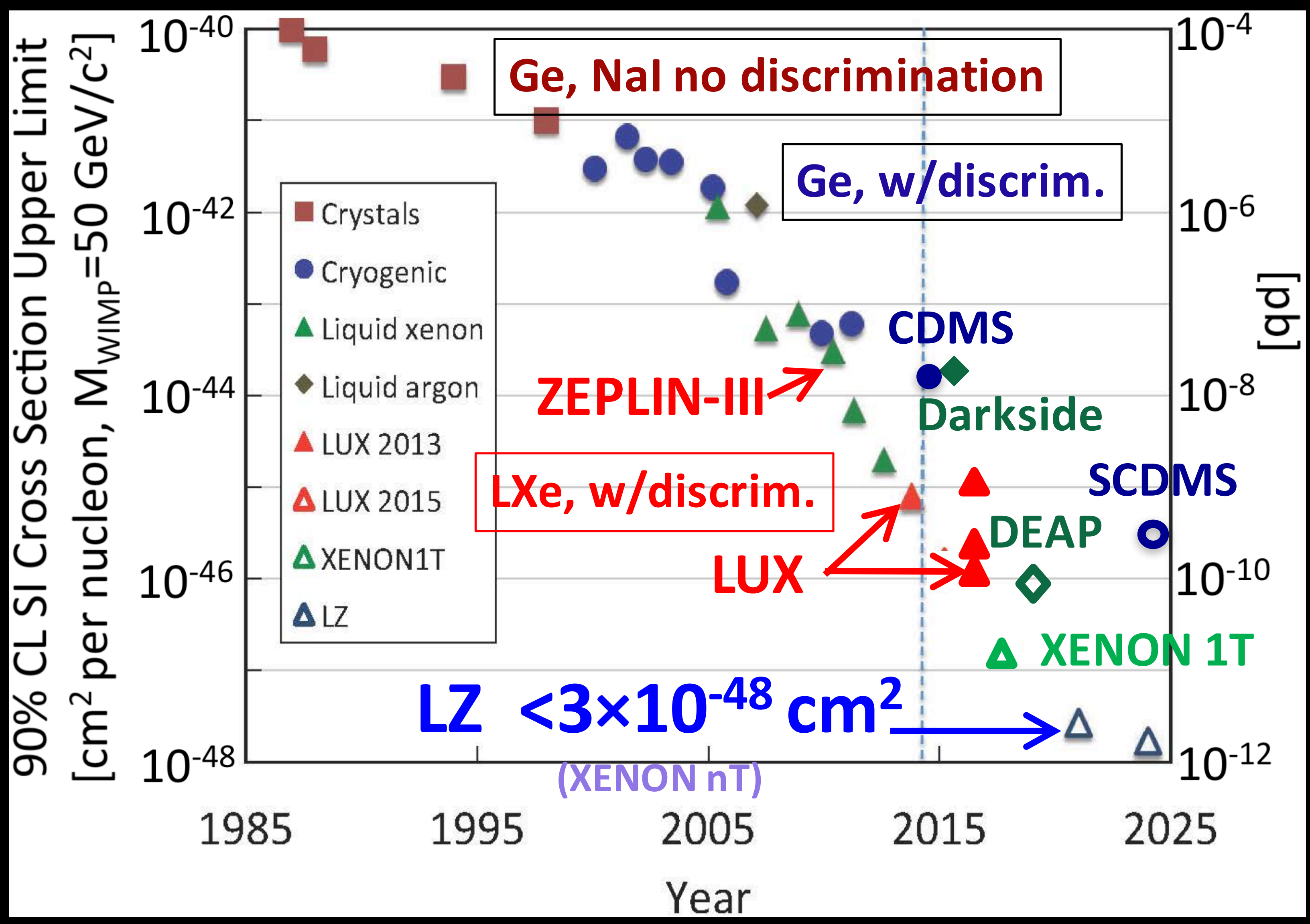


$<\sigma v>_{a n n} \approx 3 \times 10^{-26} \mathrm{~cm}^{3} \mathrm{sec}^{-1}$ $\approx \frac{\alpha^{2}}{(200 \mathrm{GeV})^{2}}$

Coupling e.g. to light quarks
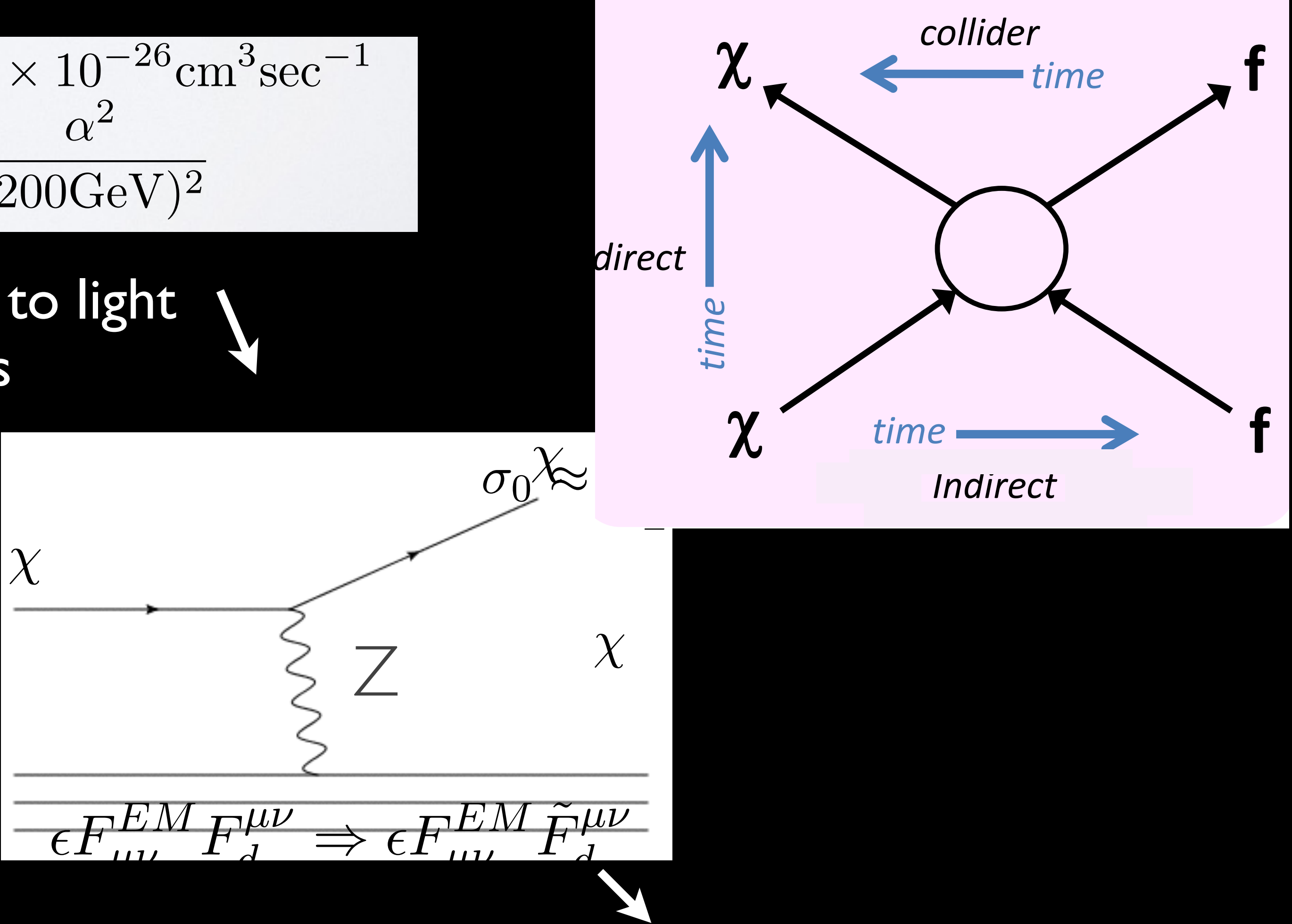

\section{Indirect}

$$
\sigma_{0} \approx \frac{G_{f}^{2} \mu^{2}}{2 \pi} \sim 10^{-39} \mathrm{~cm}^{2}
$$




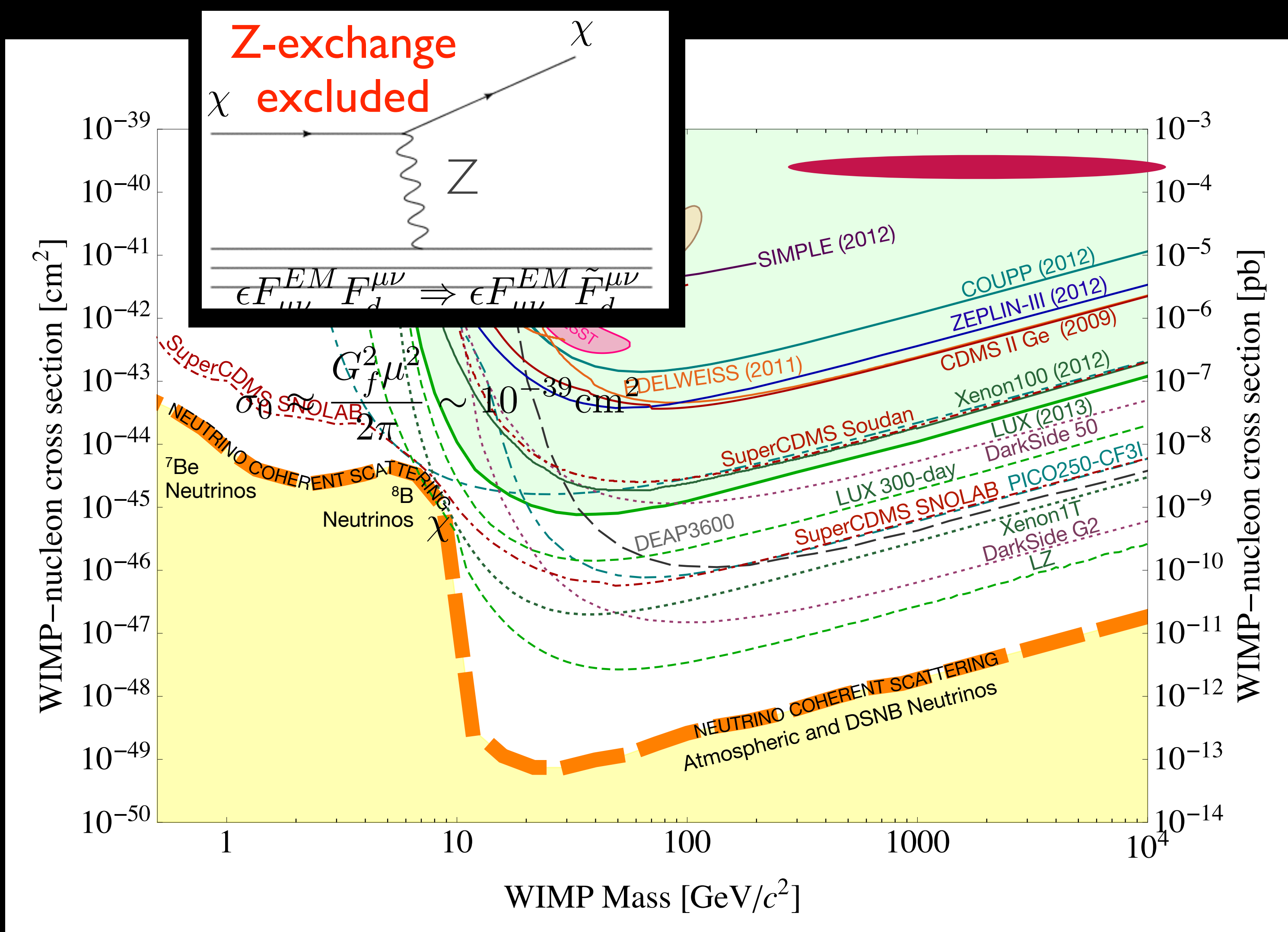




$$
\begin{aligned}
<\sigma v>_{a n n} & \approx 3 \times 10^{-26} \mathrm{~cm}^{3} \mathrm{sec}^{-1} \\
& \approx \frac{\alpha^{2}}{(200 \mathrm{GeV})^{2}}
\end{aligned}
$$

\section{Coupling proportional to} mass (e.g. via higgs)

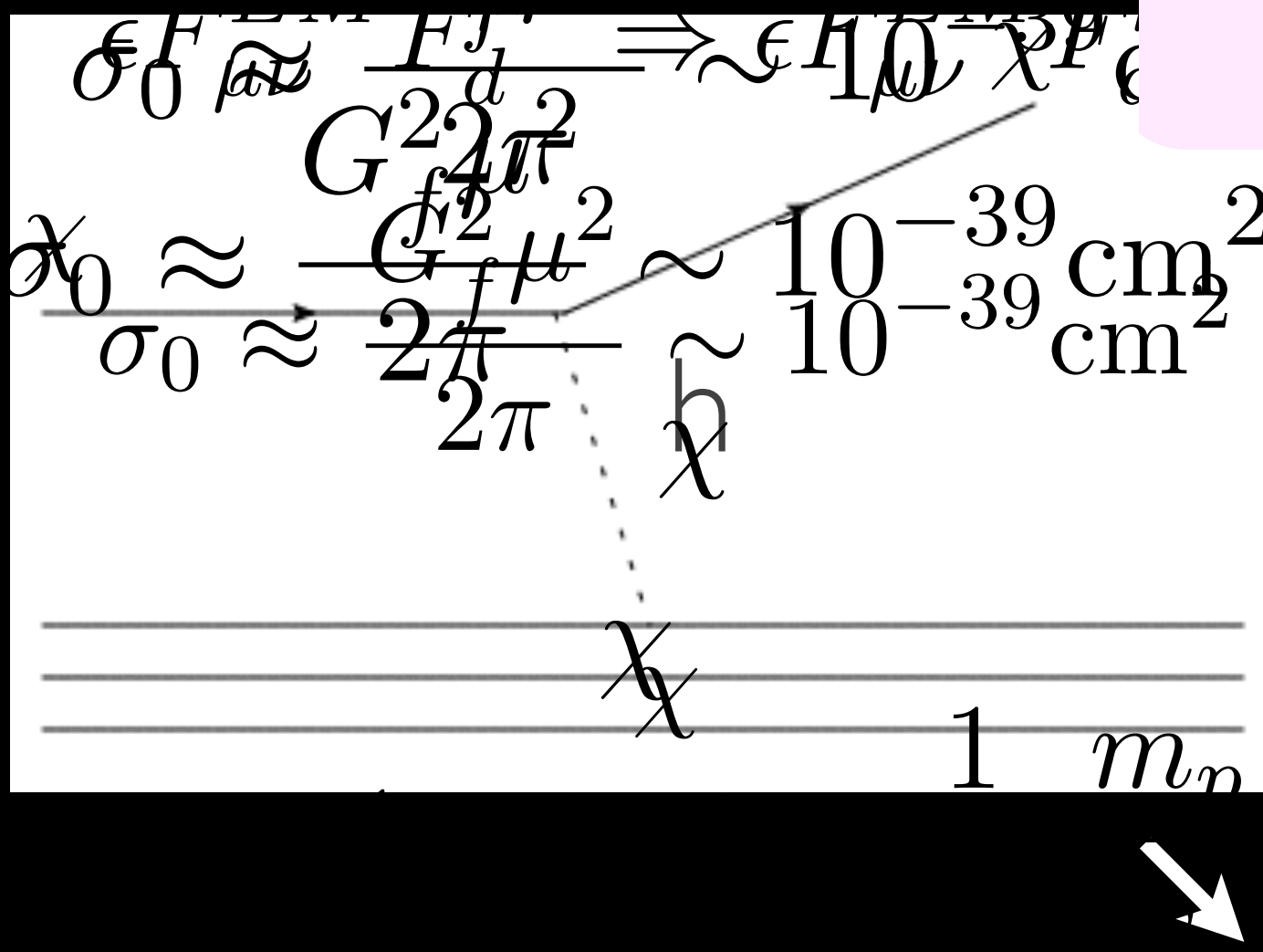

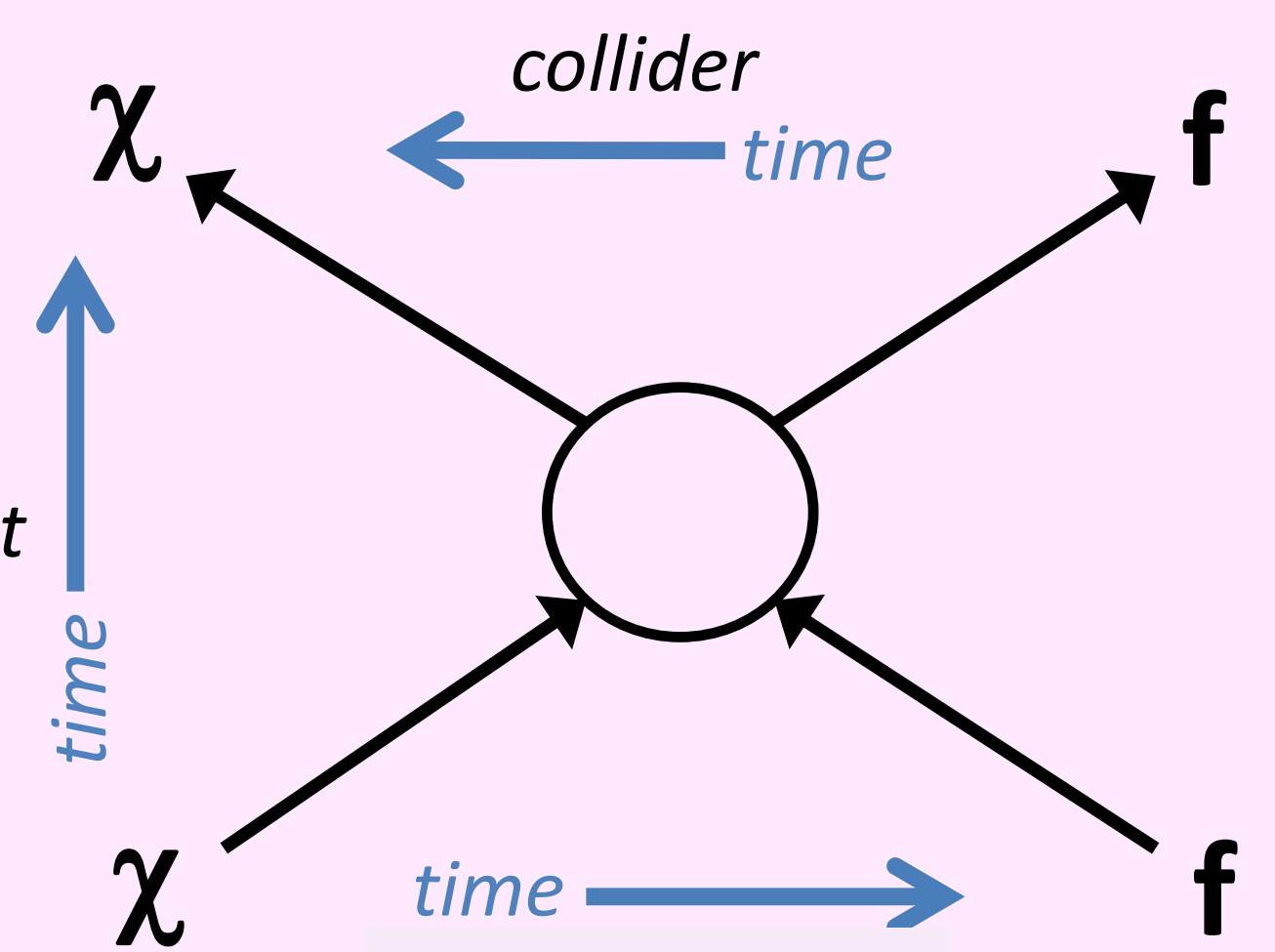

Indirect

$$
\begin{aligned}
& g \sim 1 \Rightarrow y_{p} \sim \frac{1}{\text { few }} \frac{m_{p}}{v} \\
& \sigma_{0} \sim 10^{-39} \mathrm{~cm}^{2} \times 10^{-6} \\
& \sim 10^{-45} \mathrm{~cm}^{2}
\end{aligned}
$$




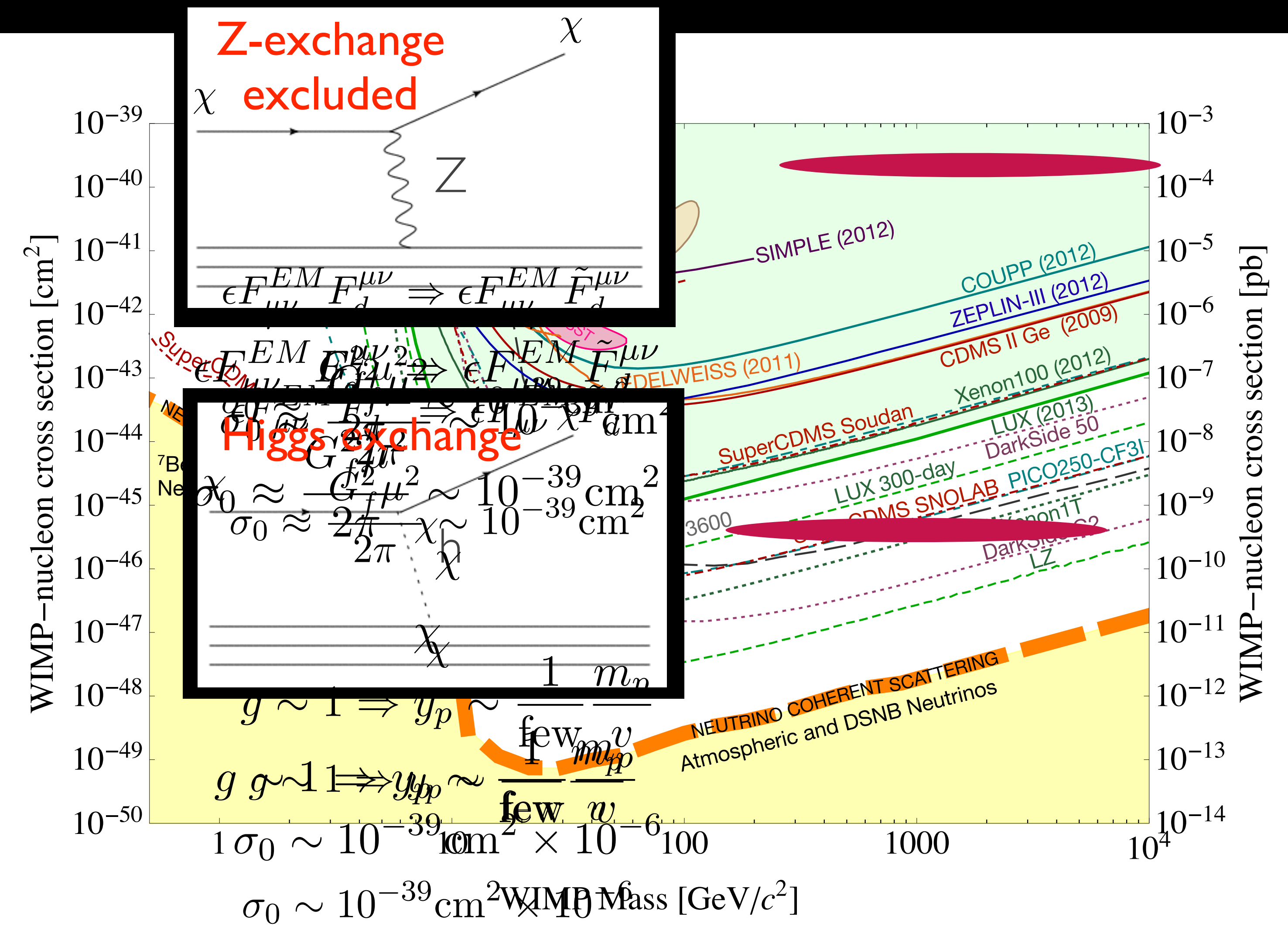

"This era will answer the question: does the dark matter couple at $\mathrm{O}(0 . \mathrm{I})$ to the Higgs boson"

N.Weiner, CIPANP 2015 


\section{The case for dark matter}

- We know it interacts gravitationally

- It is "dark" - should not interact with light or electromagnetism

- Nearly collision less

- Slow
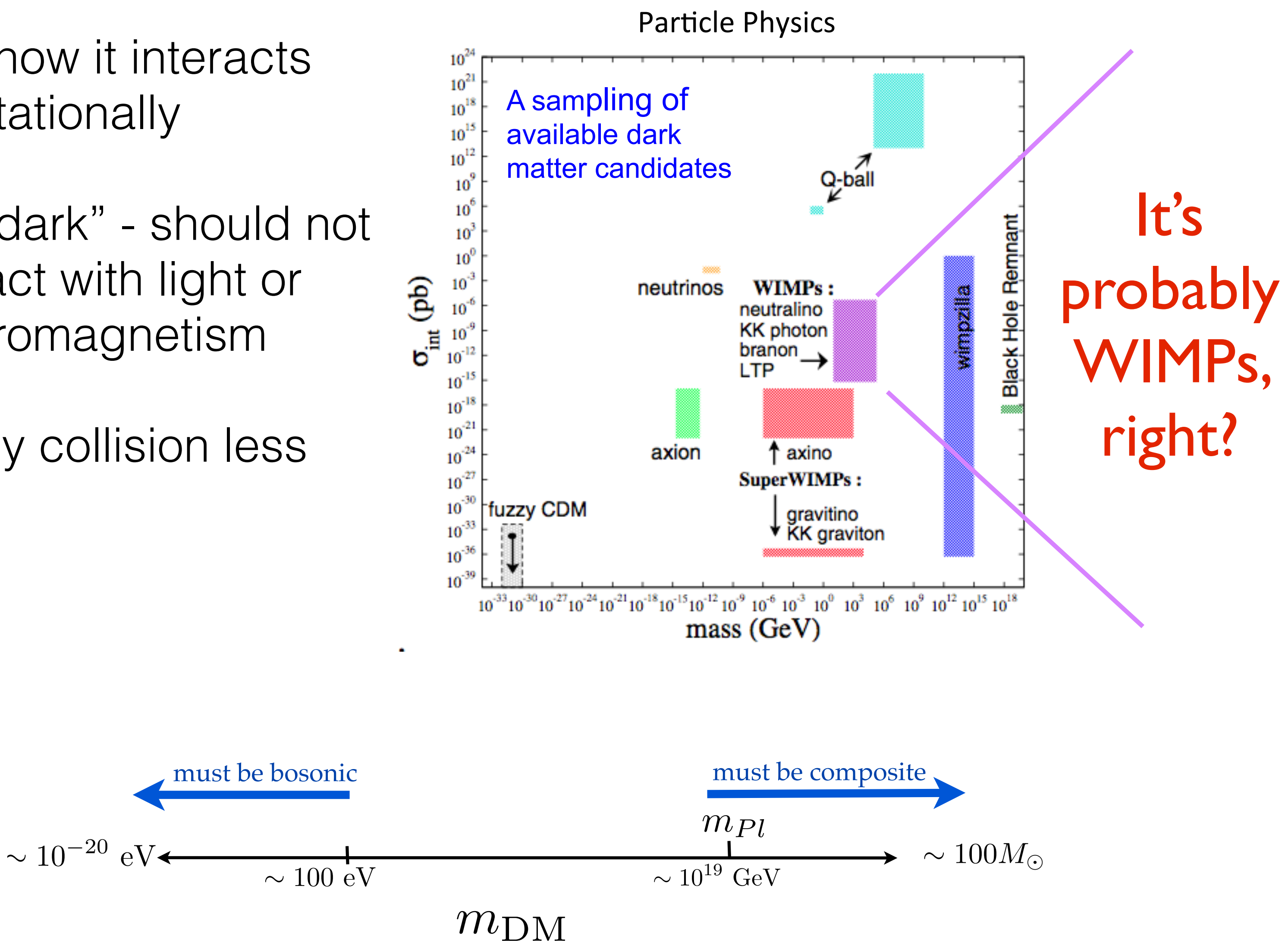


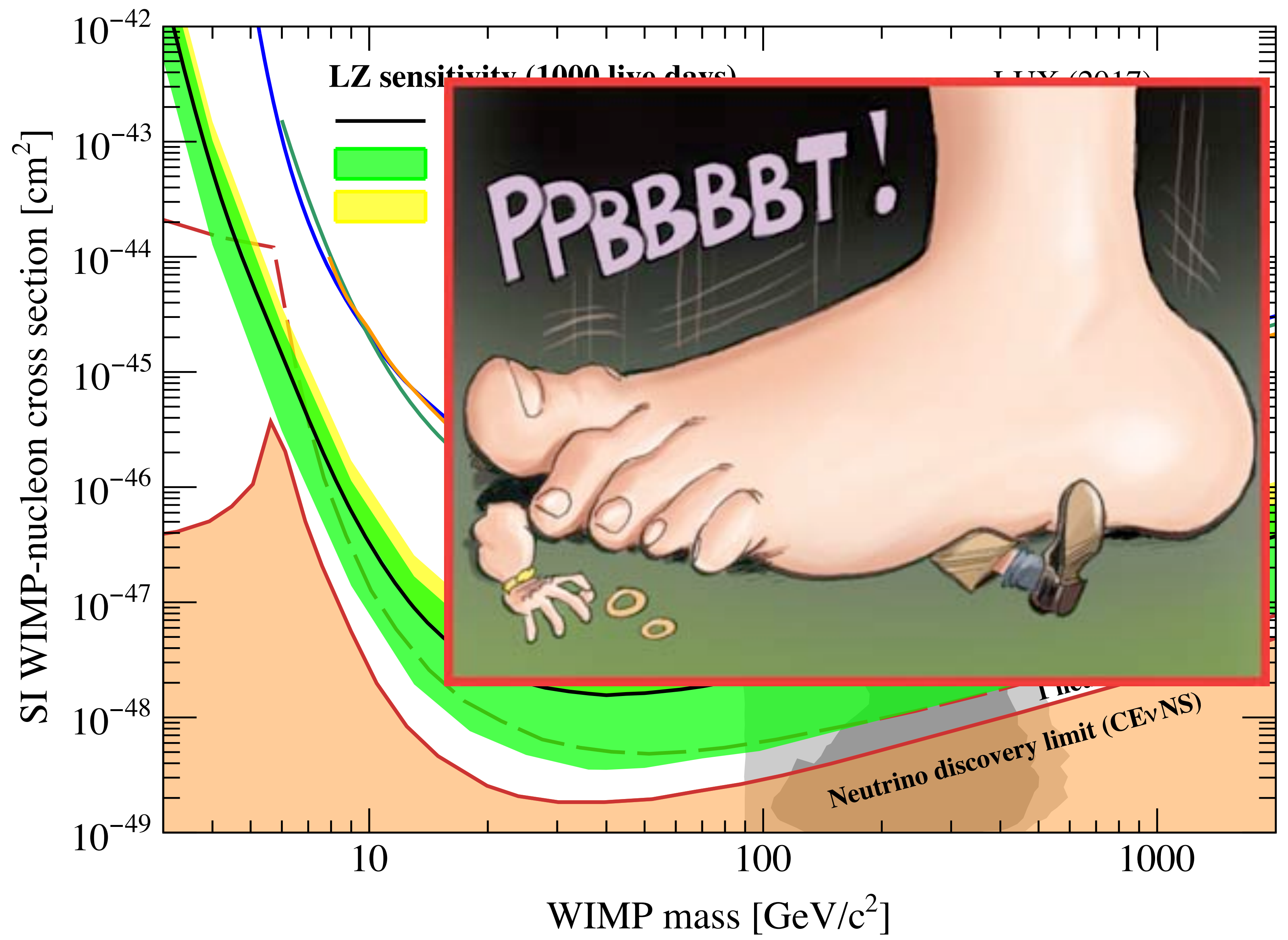




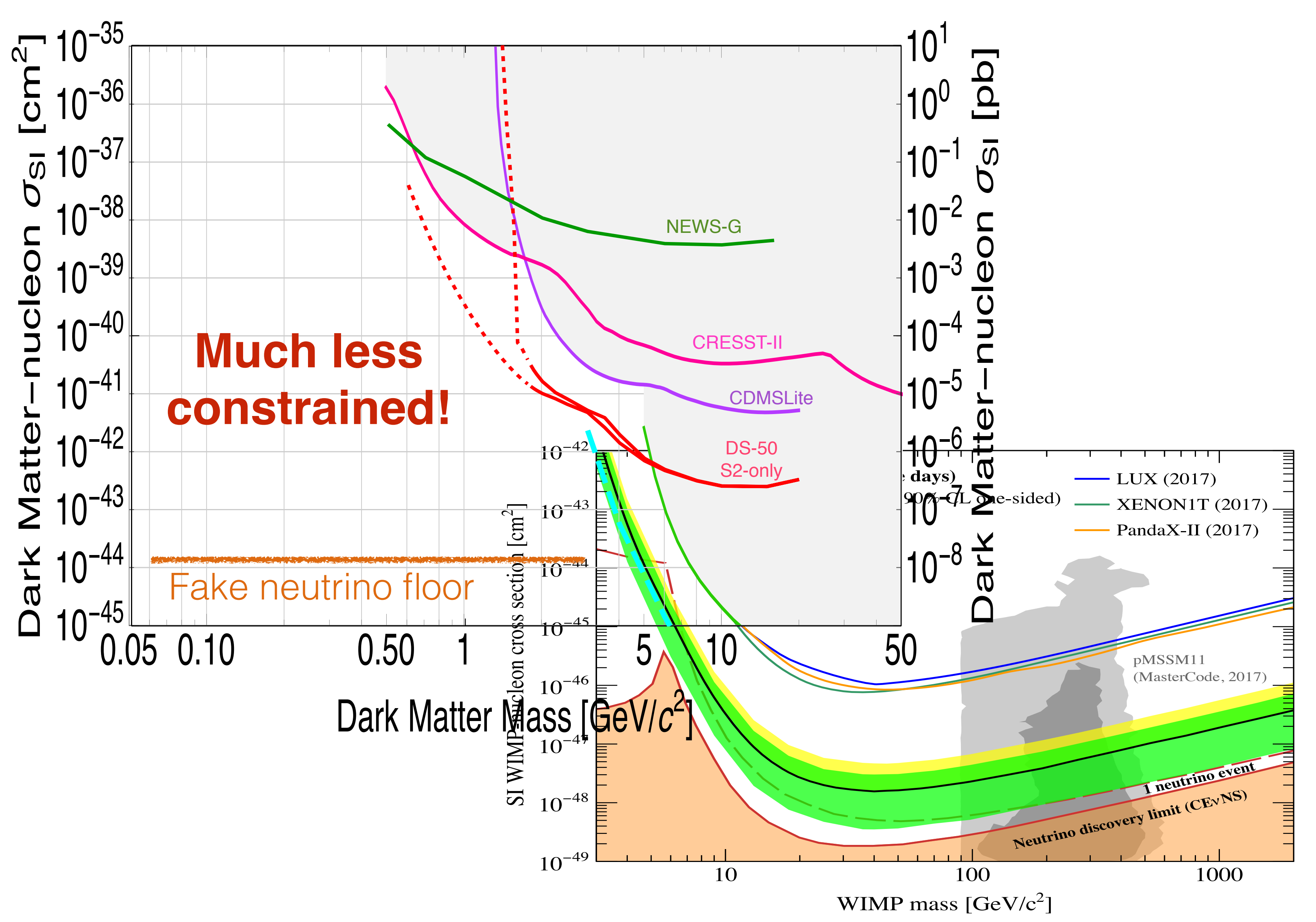




\section{DM Prognosis?}

Bad news: DM-SM interactions are not obligatory If nature is unkind, we may never know the right scale

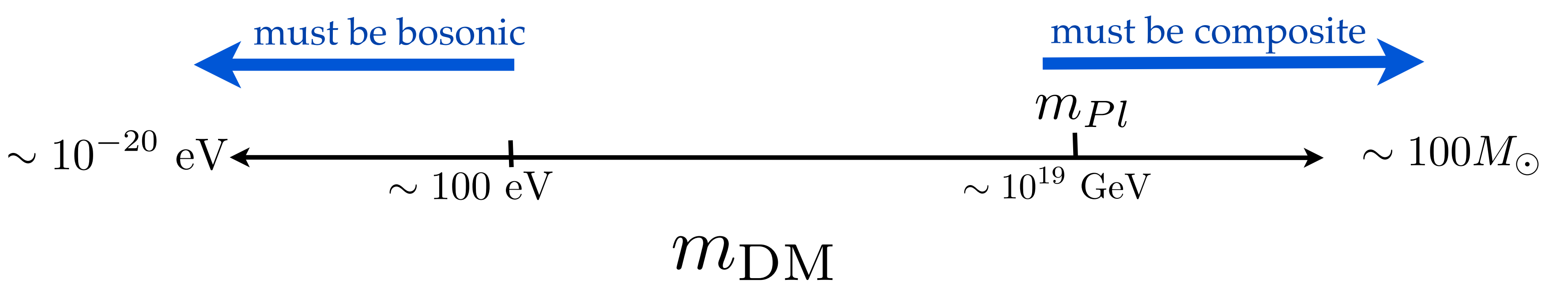

Good news: most discoverable DM candidates are in thermal equilibrium with us in the early universe 


\section{Thermal dark matter}

- "Most discoverable DM candidates are in thermal equilibrium" - G. Krnjaic

- If we can detect it, it's likely that it was in equilibrium (e.g. interacted enough)

- Thermal dark matter has minimum annihilation rate (to set relic density)

- Doesn't care about initial conditions (washed out by thermal bath) - makes modeling easier

- Limited viable mass range (to a range that is basically within reach)

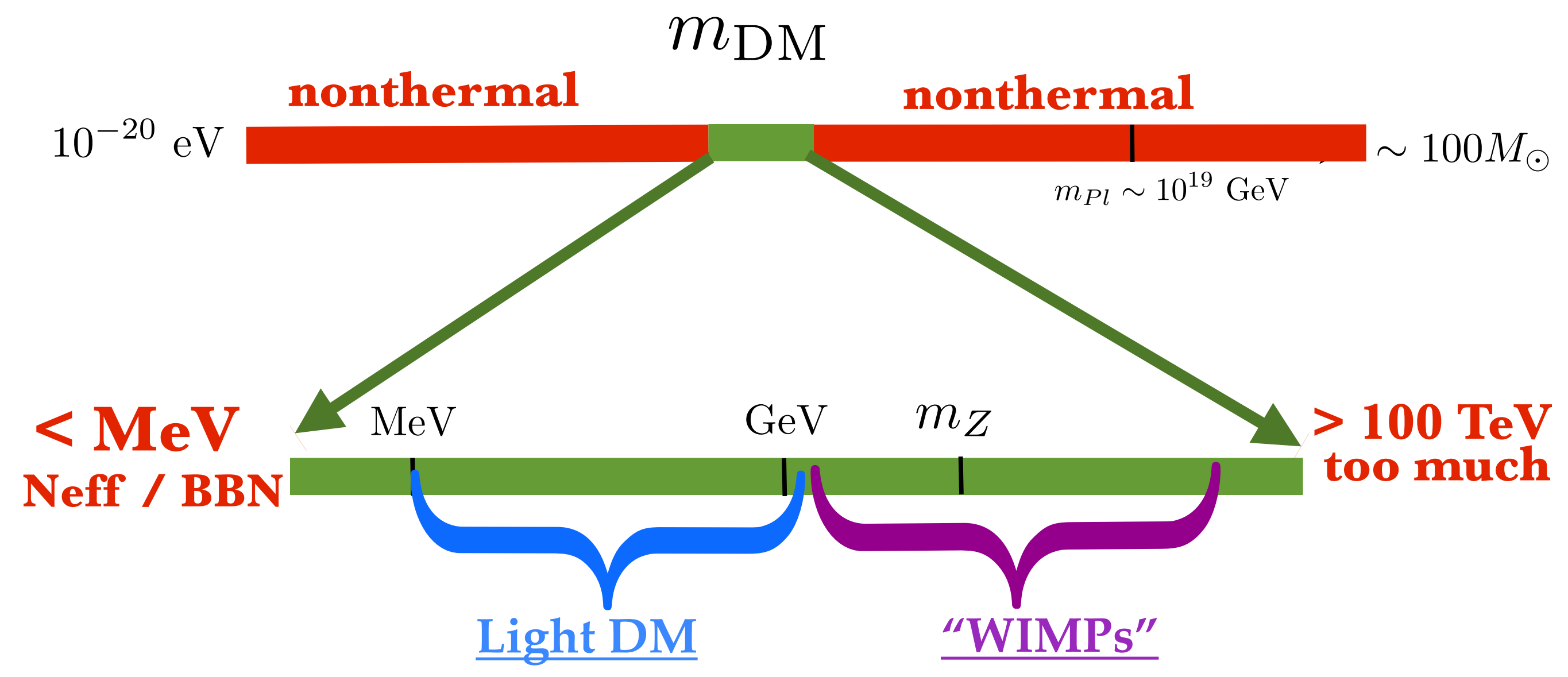




\section{Thermal dark matter}

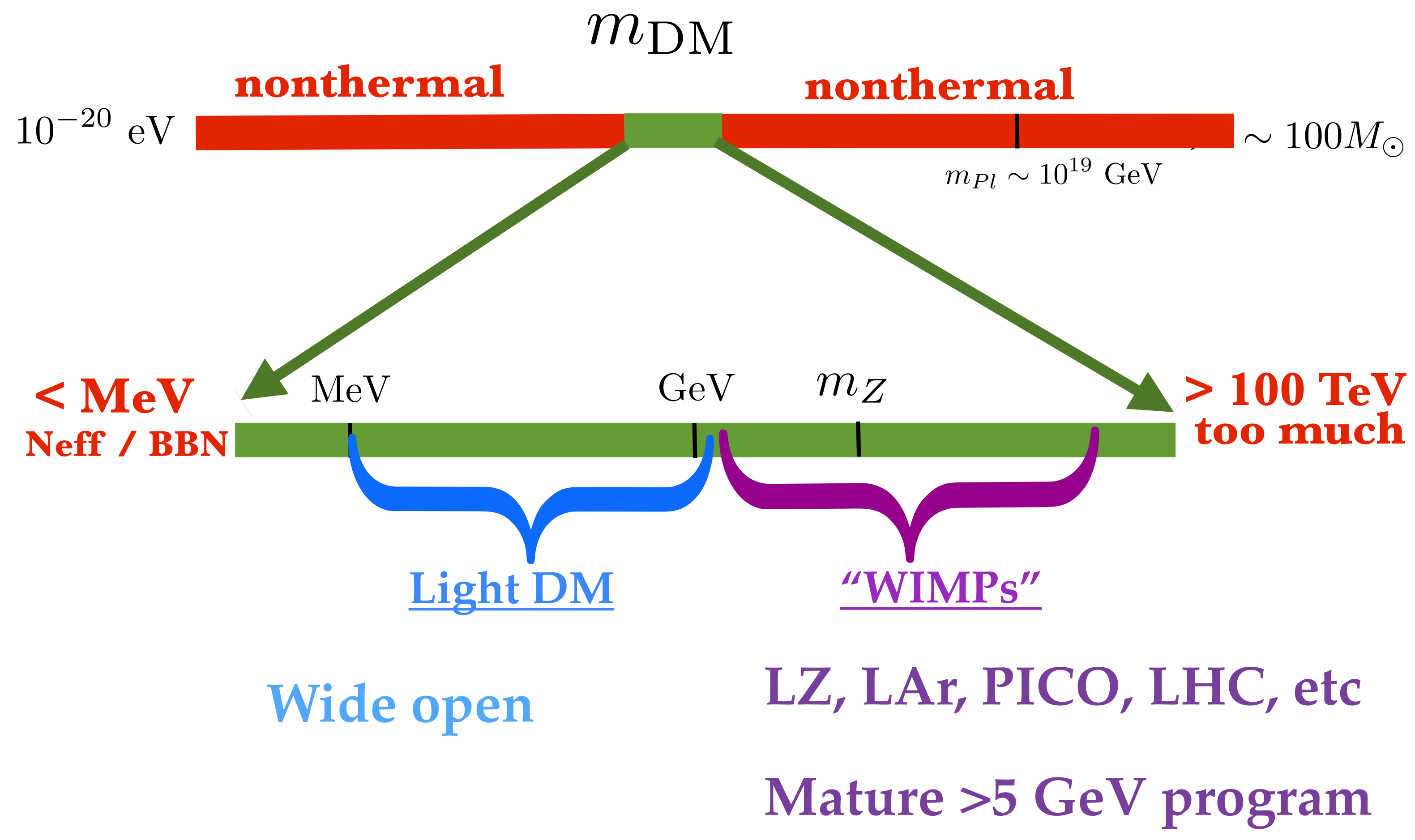




\section{Are there actual candidates?}

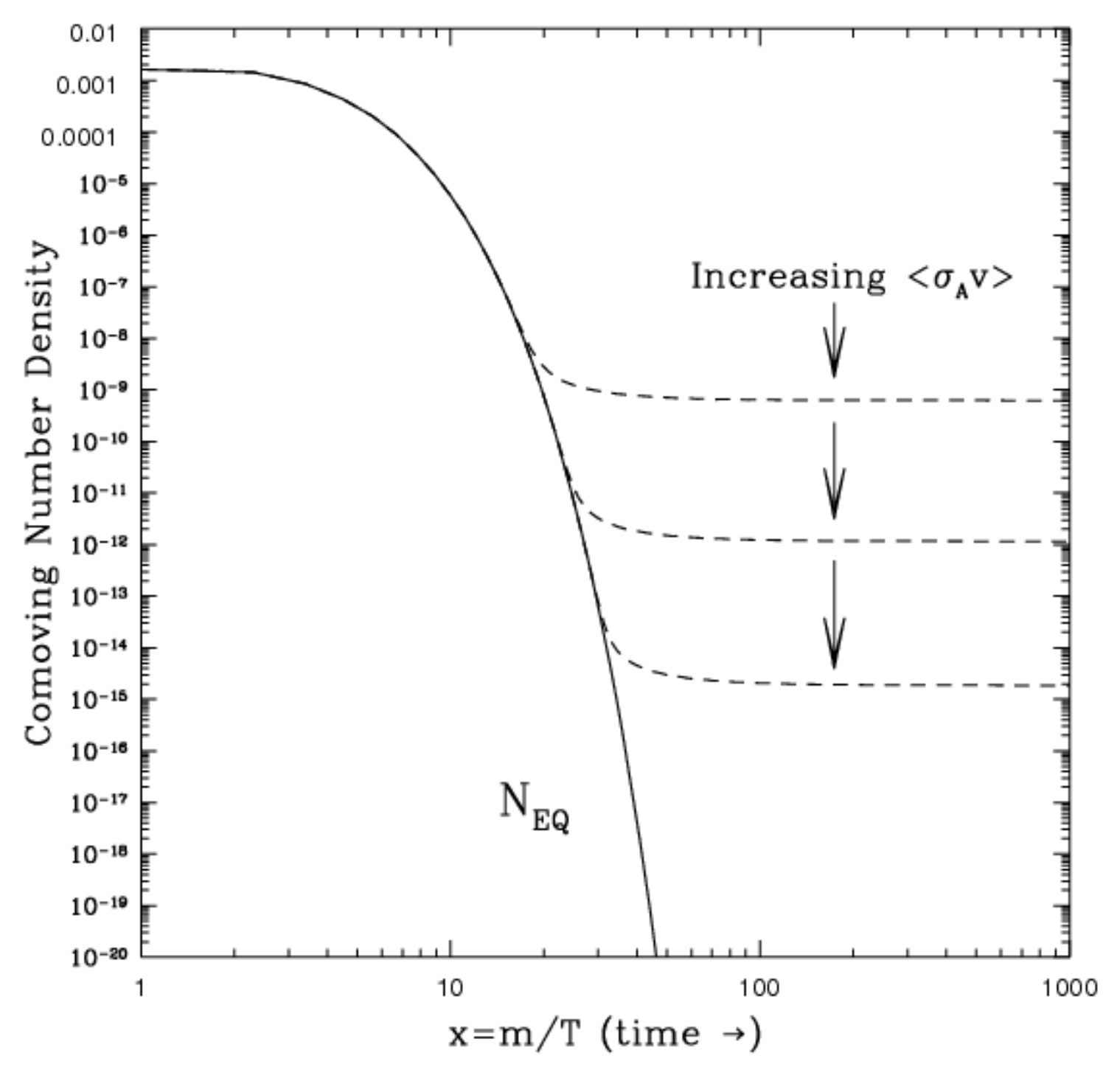

- Annihilation cross section needed for the relic abundance

$$
<\sigma v>_{a n n} \approx 3 \times 10^{-26} \mathrm{~cm}^{3} \mathrm{sec}^{-1}
$$

- New weak scale particle has to be heavier than a few GeV

- Lee and Weinberg, PRL 39 (1977) 165-168

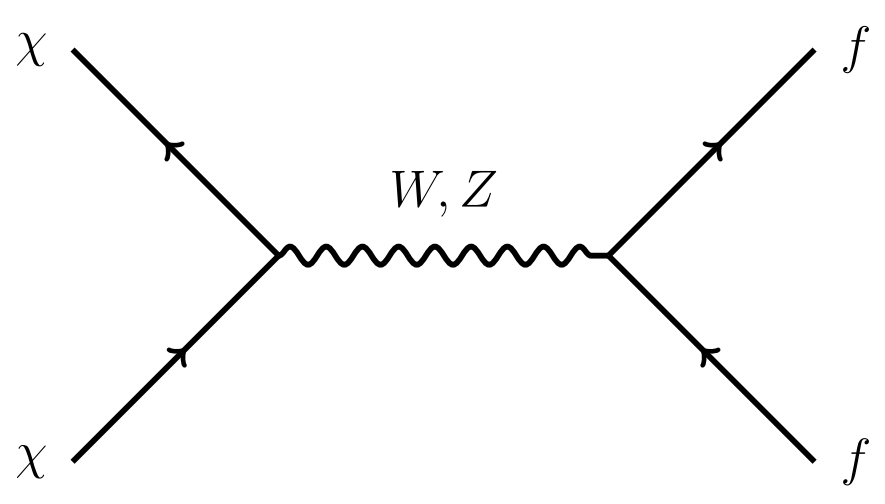

$$
\sigma v \sim \frac{\alpha^{2} m_{\chi}^{2}}{m_{Z}^{4}} \sim 10^{-29} \mathrm{~cm}^{3} \mathrm{~s}^{-1}\left(\frac{m_{\chi}}{\mathrm{GeV}}\right)^{2}
$$




\section{Are there actual candidates?}

- Light dark matter needs new forces (although we might already be there in canonical WIMP dark matter anyway)

- Asymmetric DM

- Secluded DM

- Forbidden DM

- SIMP

- ELDER

- Freeze in models
US Cosmic Visions: New Ideas in Dark Matter 2017 :

Community Report

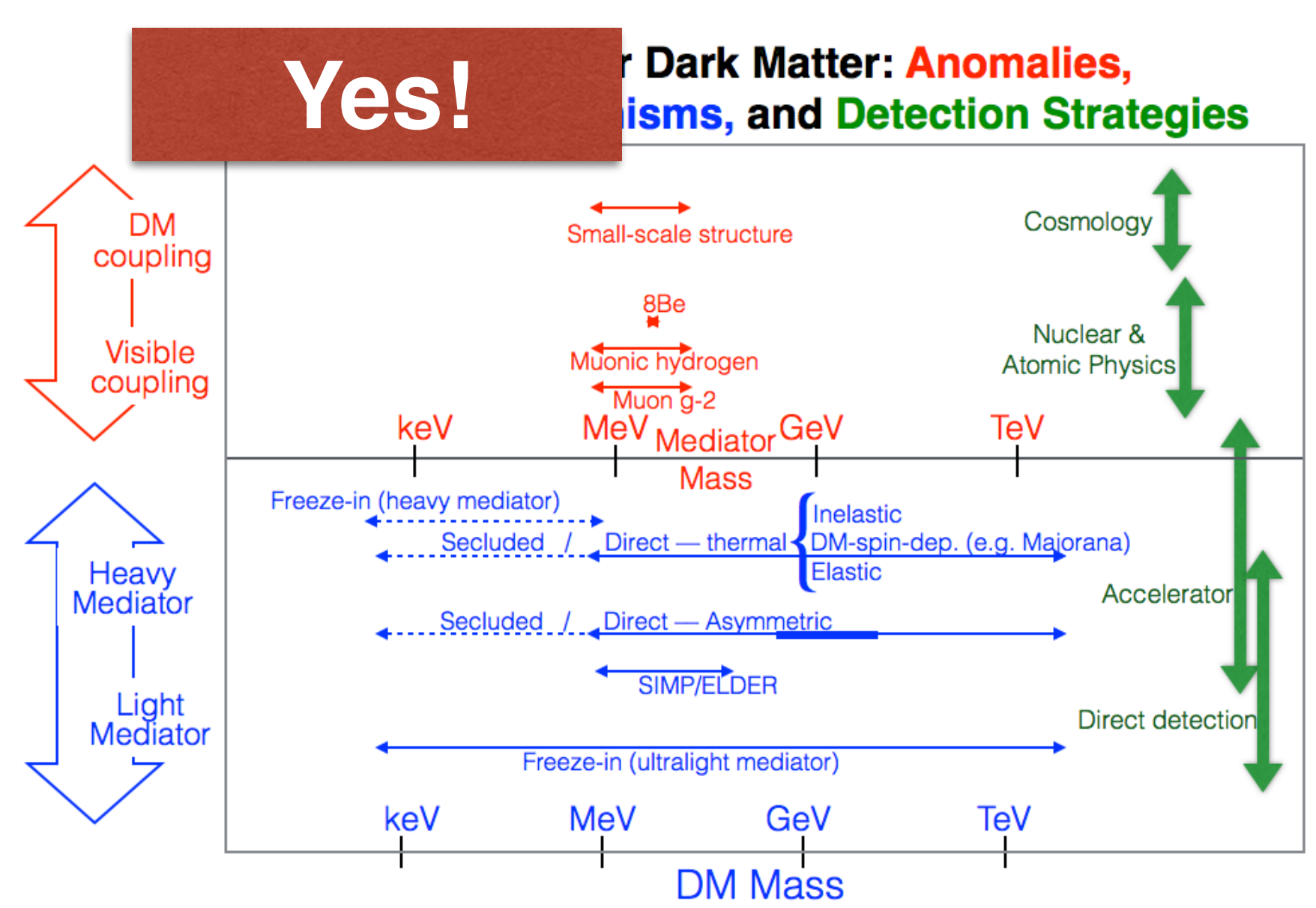


What do you need for low mass?

$$
\frac{d R}{d Q}=\frac{\rho_{0}}{m_{\chi}} \times \frac{\sigma_{0} A^{2}}{2 m_{p}^{2}} \times F^{2}(Q) \times \int_{v_{m}}^{v_{e s c}} \frac{f(v)}{v} d v
$$

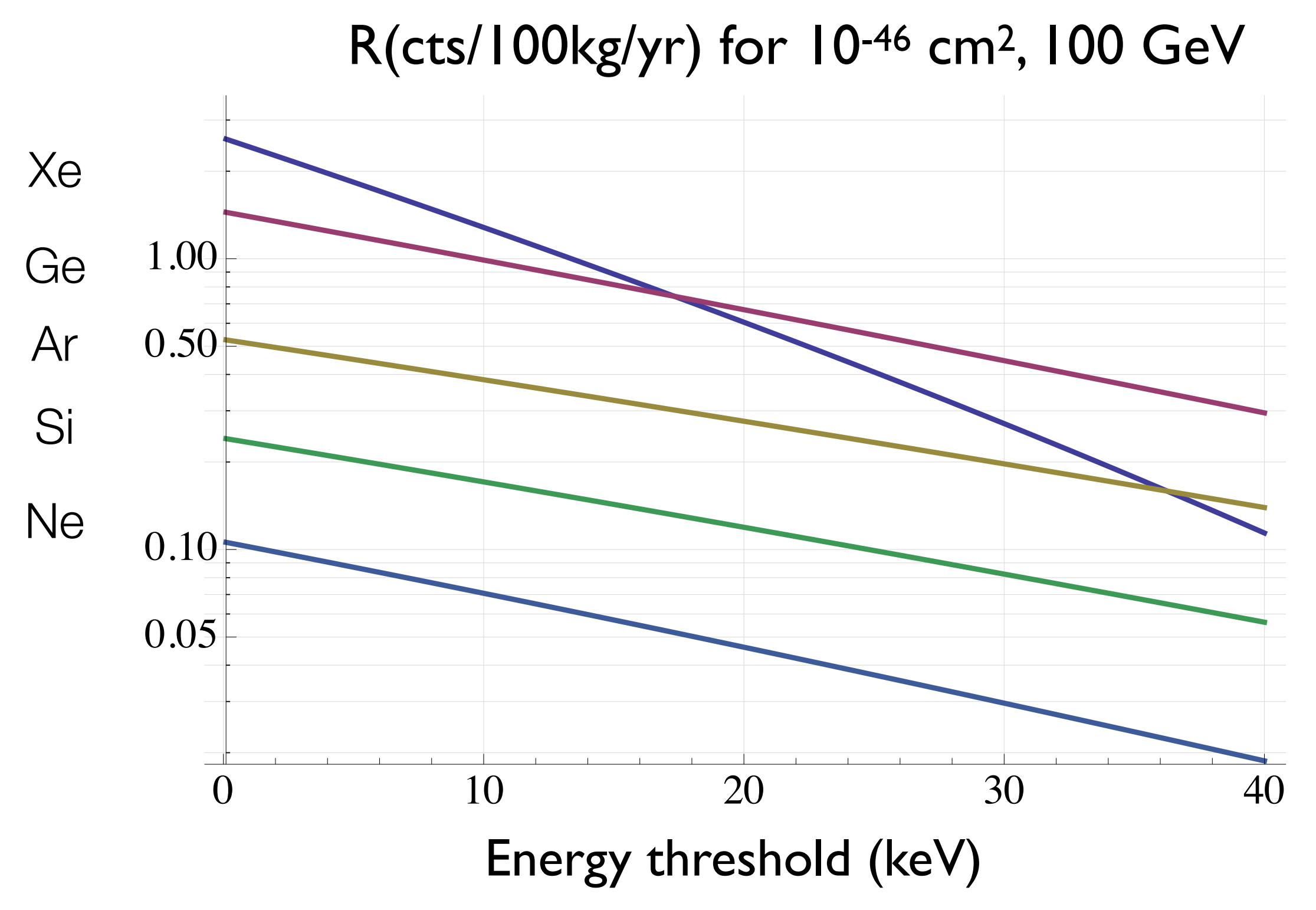


What do you need for low mass?

$$
\frac{d R}{d Q}=\frac{\rho_{0}}{m_{\chi}} \times \frac{\sigma_{0} A^{2}}{2 m_{p}^{2}} \times F^{2}(Q) \times \int_{v_{m}}^{v_{e s c}} \frac{f(v)}{v} d v
$$

\section{$\mathrm{R}\left(\mathrm{cts} / 10 \mathrm{~kg} / \mathrm{yr}\right.$ ) for $10^{-45} \mathrm{~cm}^{2}, 10 \mathrm{GeV}$}

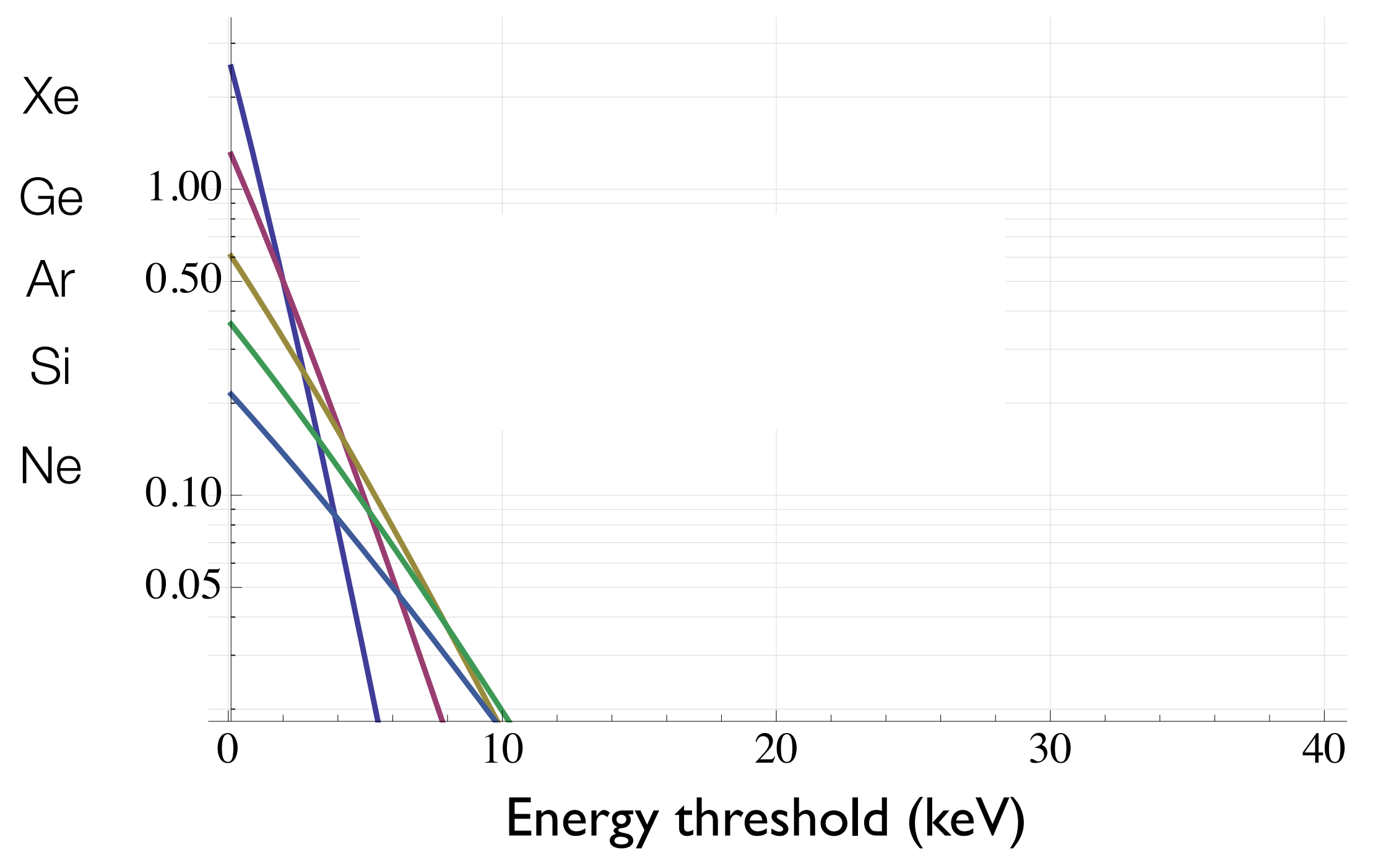


What do you need for low mass?

$$
\begin{gathered}
\frac{d R}{d Q}=\frac{\rho_{0}}{m_{\chi}} \times \frac{\sigma_{0} A^{2}}{2 m_{p}^{2}} \times F^{2}(Q) \times \int_{v_{m}}^{v_{e s c}} \frac{f(v)}{v} d v \\
v_{m}=\sqrt{Q m_{N} / 2 m_{r}^{2}} \quad v_{e s c}=544 \mathrm{~km} / \mathrm{s}(\text { current value }) \\
m_{N} \text { is mass of nucleus } \quad m_{r}=\frac{m_{N} m_{\chi}}{m_{N}+m_{\chi}}
\end{gathered}
$$

- Low threshold

- Low mass target (for better kinematic match to the dark matter mass)

- For given $Q, v_{m}$ is minimized when $m_{n}=m_{\chi}$ 


\section{What do you need for low mass?}

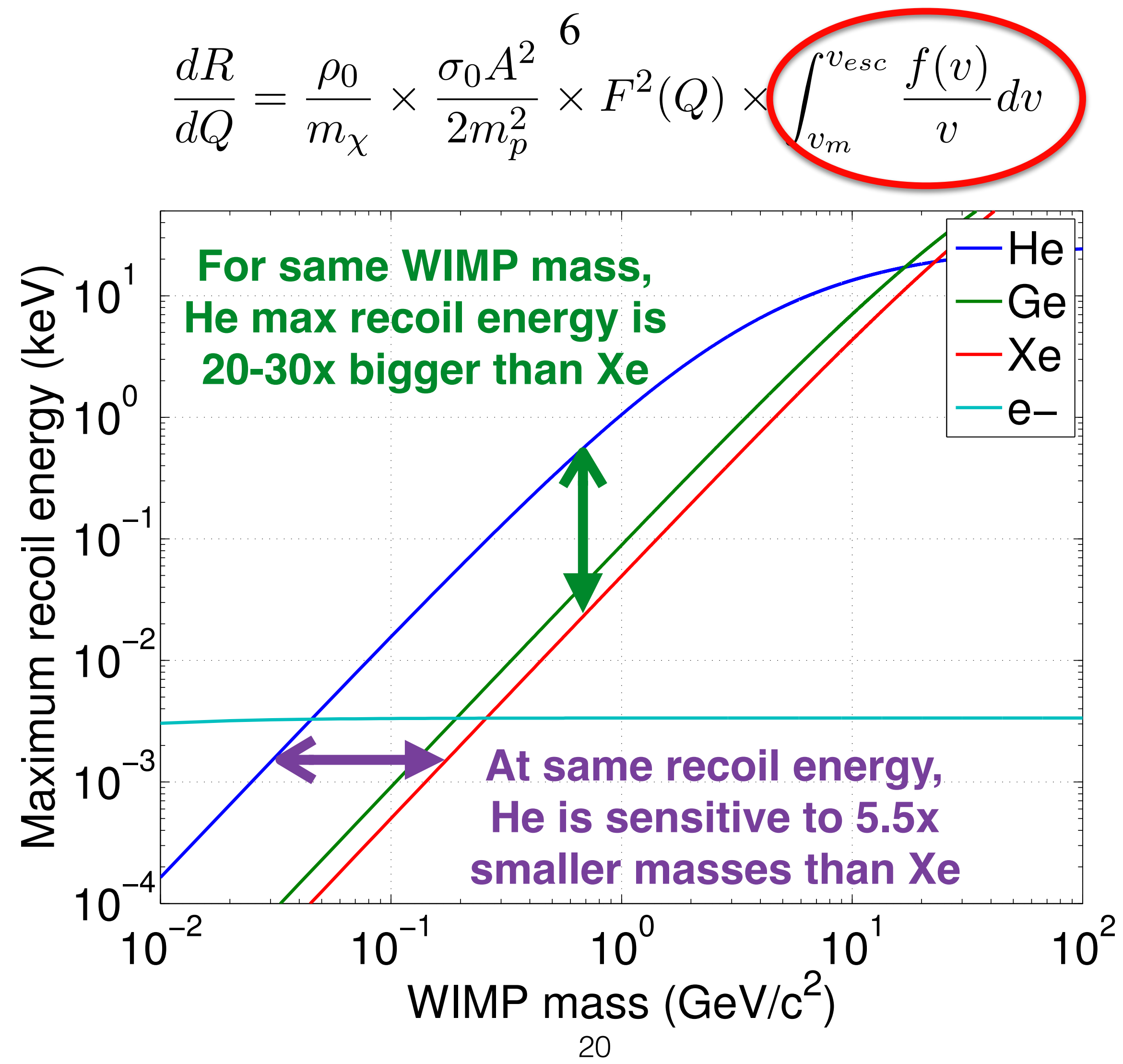




\section{Light targets less sensitive to halo uncertainty}

$$
\frac{d R}{d Q}=\frac{\rho_{0}}{m_{\chi}} \times \frac{\sigma_{0} A^{2}}{2 m_{p}^{2}} \times F^{2}(Q) \cup \int_{v_{m}}^{v_{e s c}} \frac{f(v)}{v} d v
$$
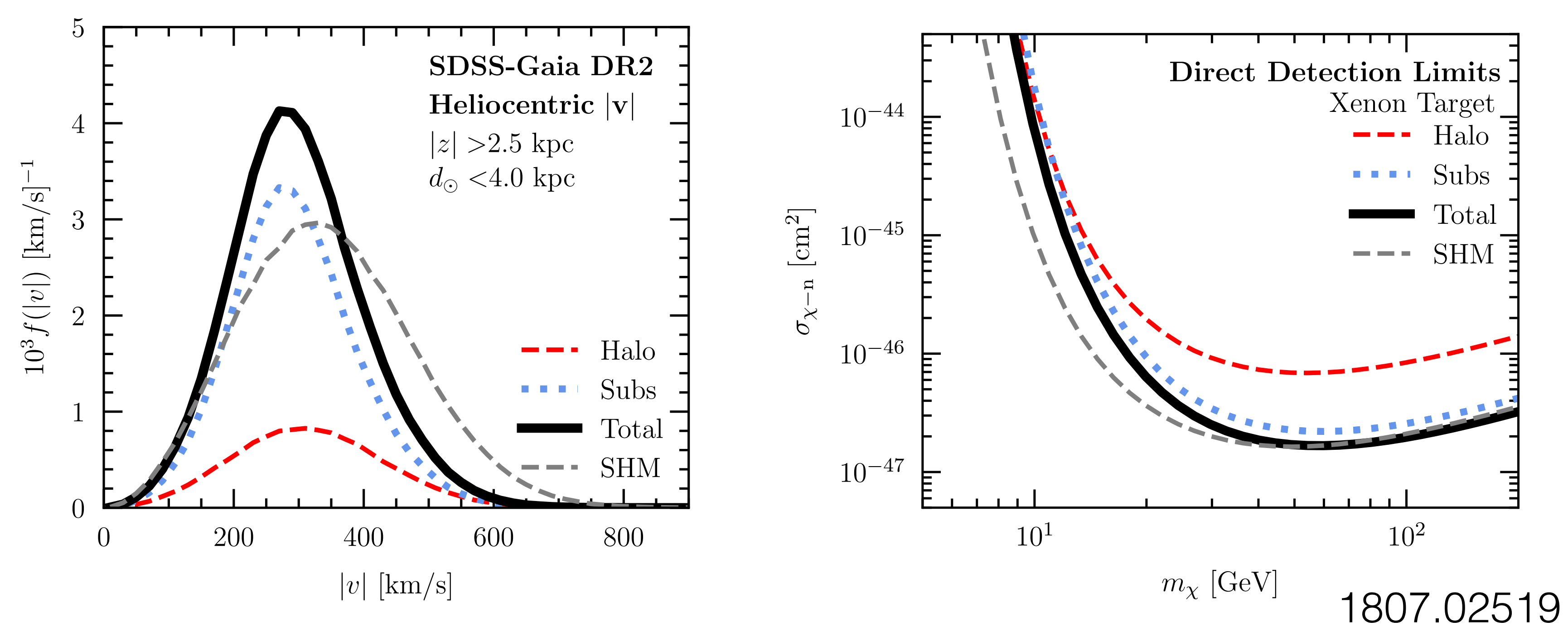


\section{Light targets less sensitive to halo uncertainty}

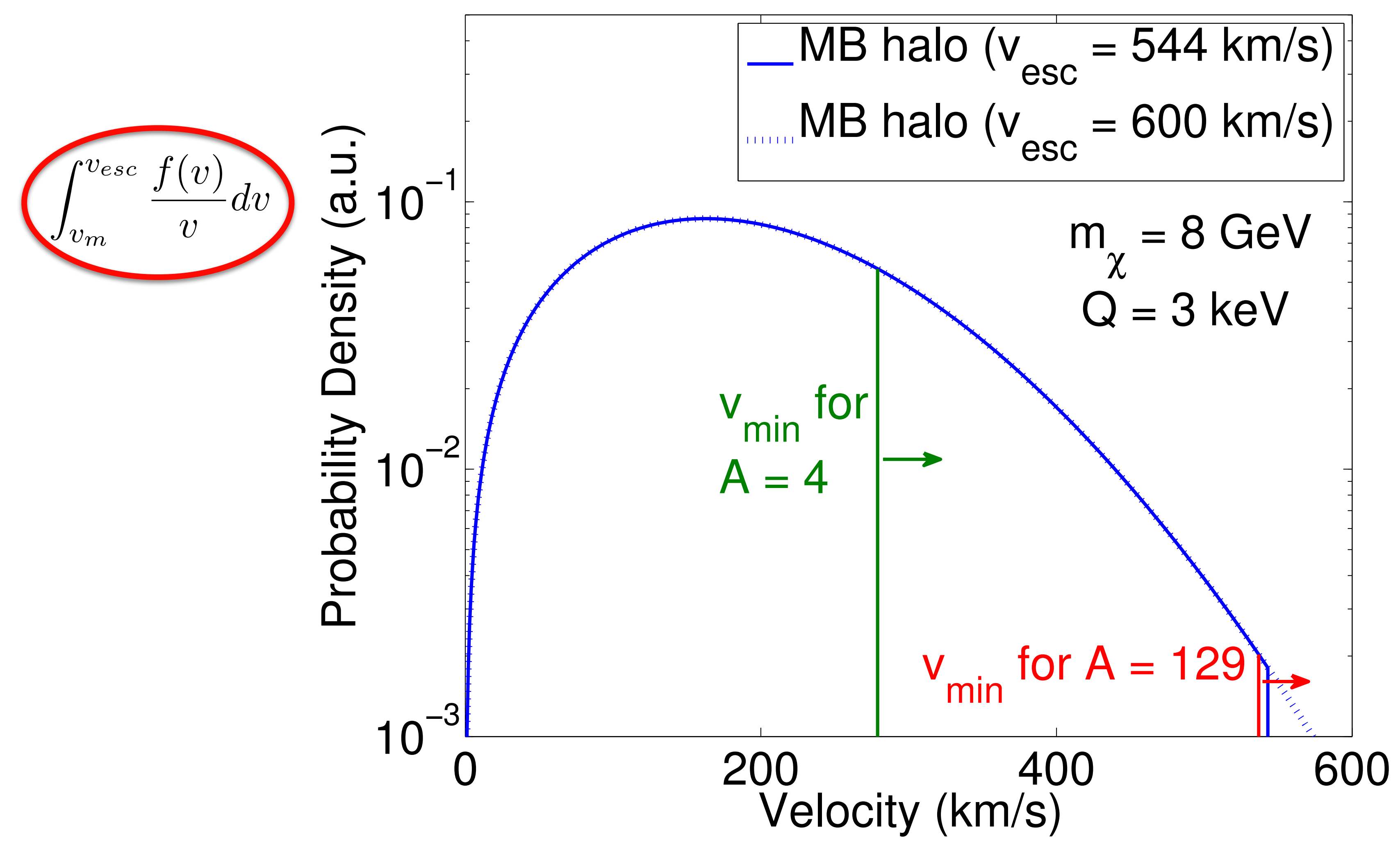




\section{What don't you need for low mass?}

- A lot of mass

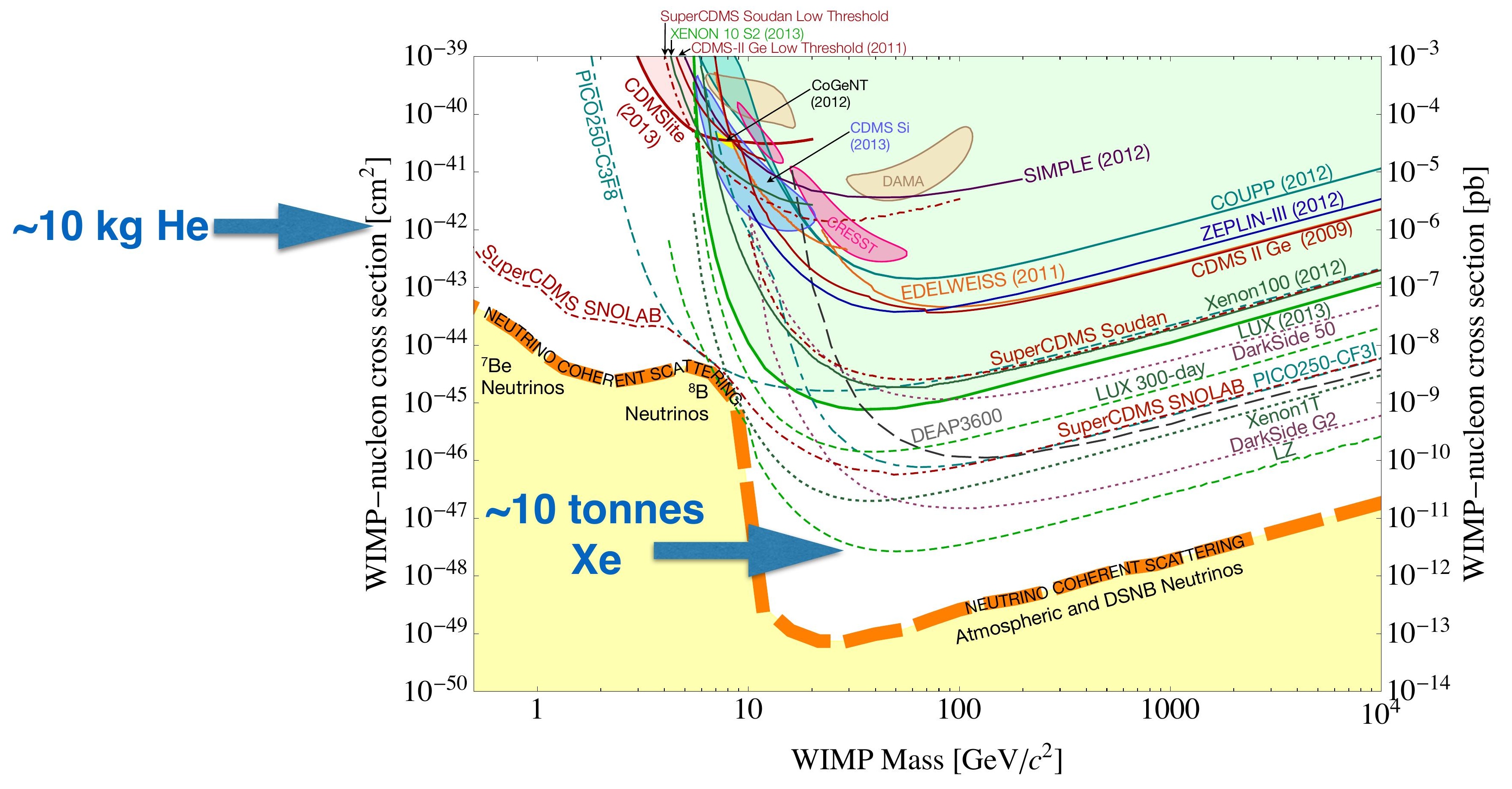




\section{LUX-Zeplin (LZ)}

- 7 tonne active LXe TPC

- Heavy target

- Excellent self shielding

- Good discrimination

- Low threshold (<3 keV)

- Huge effort to make it clean and low background

- >30 institutions, 200 people

- Now under construction in Lead, SD

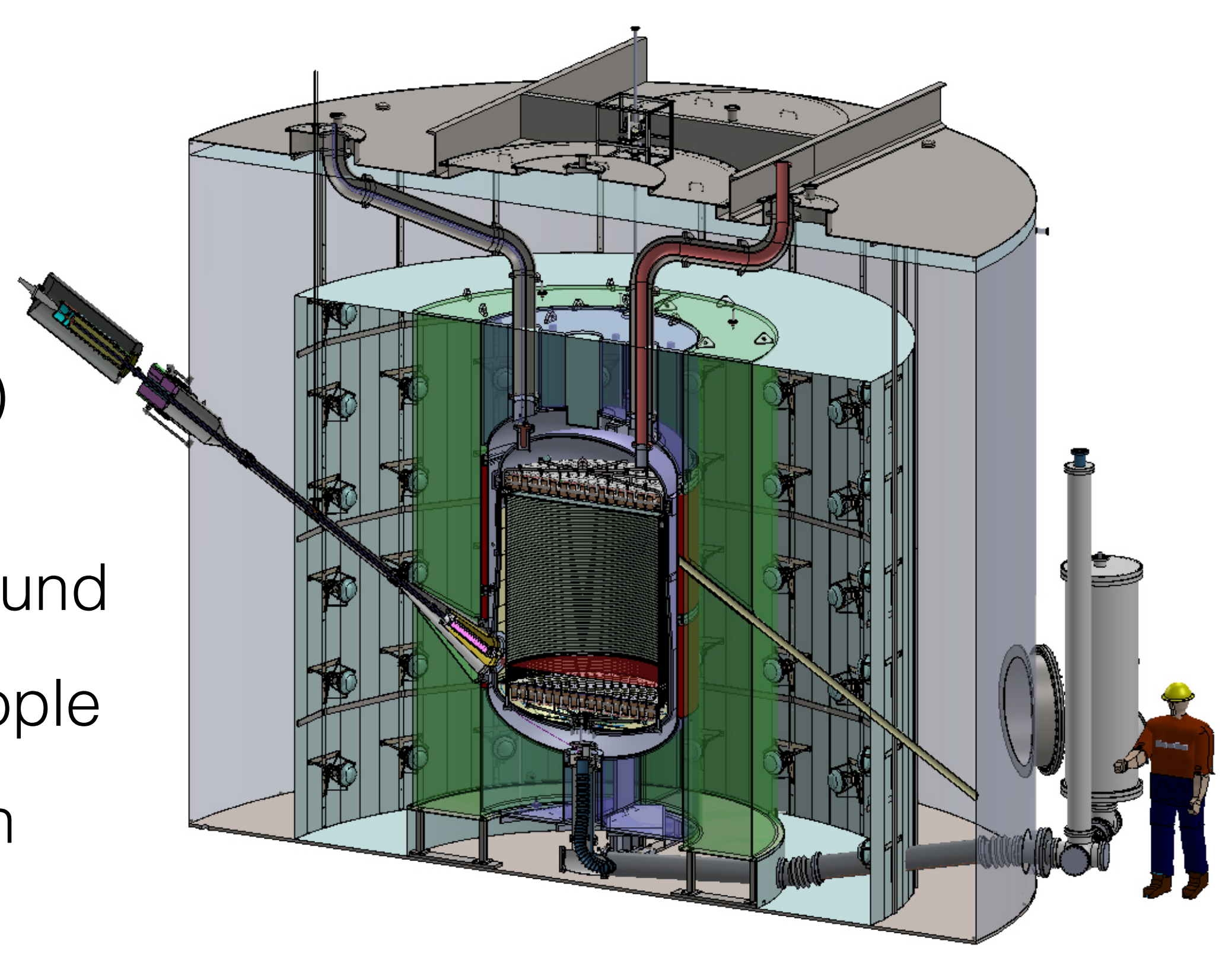




\section{Two phase Xenon TPCs}

- Interaction in the xenon creates:

- Scintillation light ( 10 ns)

- called SI

- ionization electrons

- Electrons drift through electric field to liquid/gas surface

- Extracted into gas and accelerated creating proportional scintillation light - called S2

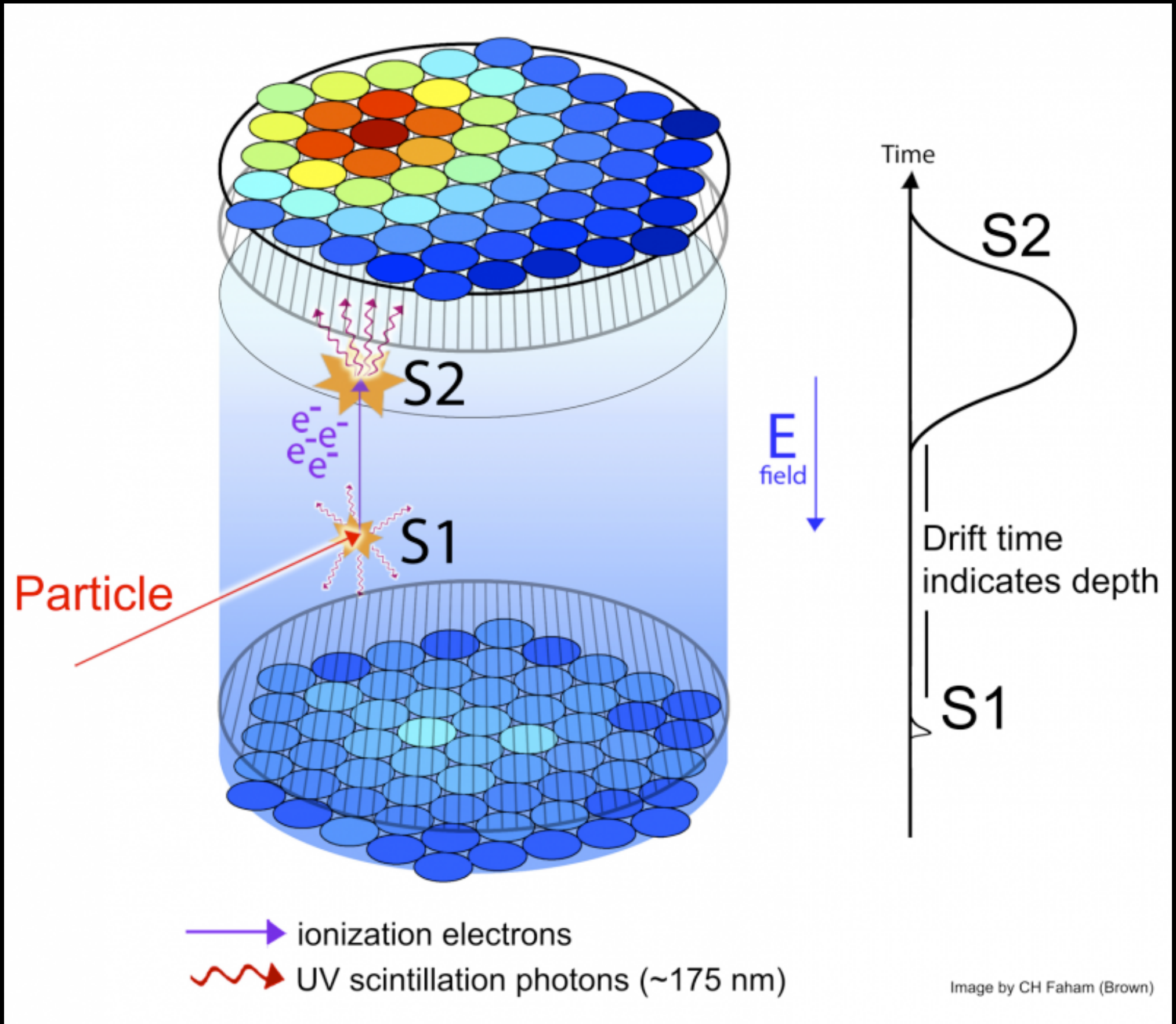




\section{Two phase Xenon TPCs}

- Excellent 3D reconstruction ( $\sim \mathrm{mm})$

- Z position from SI-S2 timing

- XY position from hit pattern of S2 light

- Allows for self shielding, rejection of edge events

- Ratio of charge (S2) to light (SI) gives particle ID

- Better than $99.5 \%$
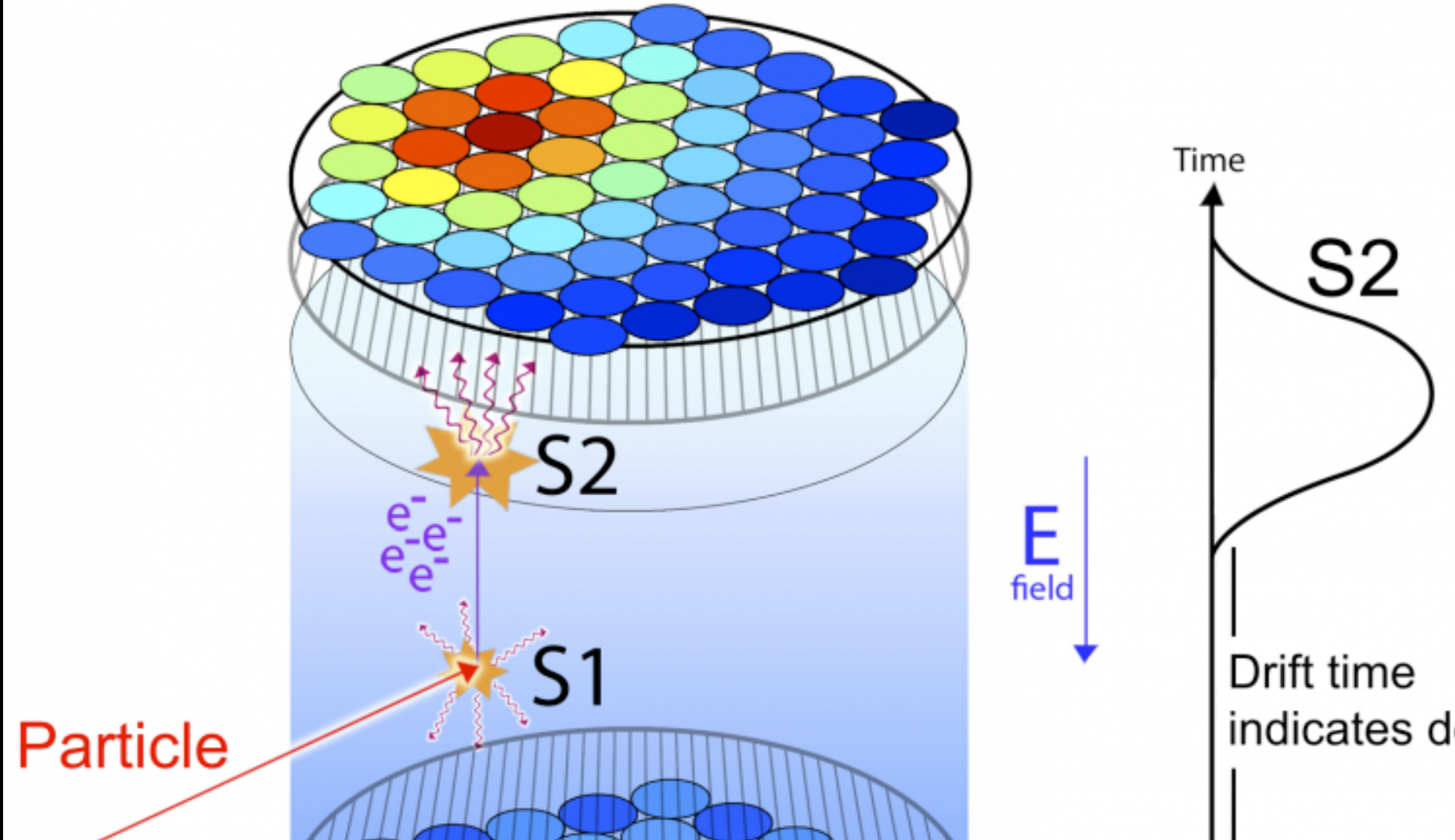

Drift time indicates depth r
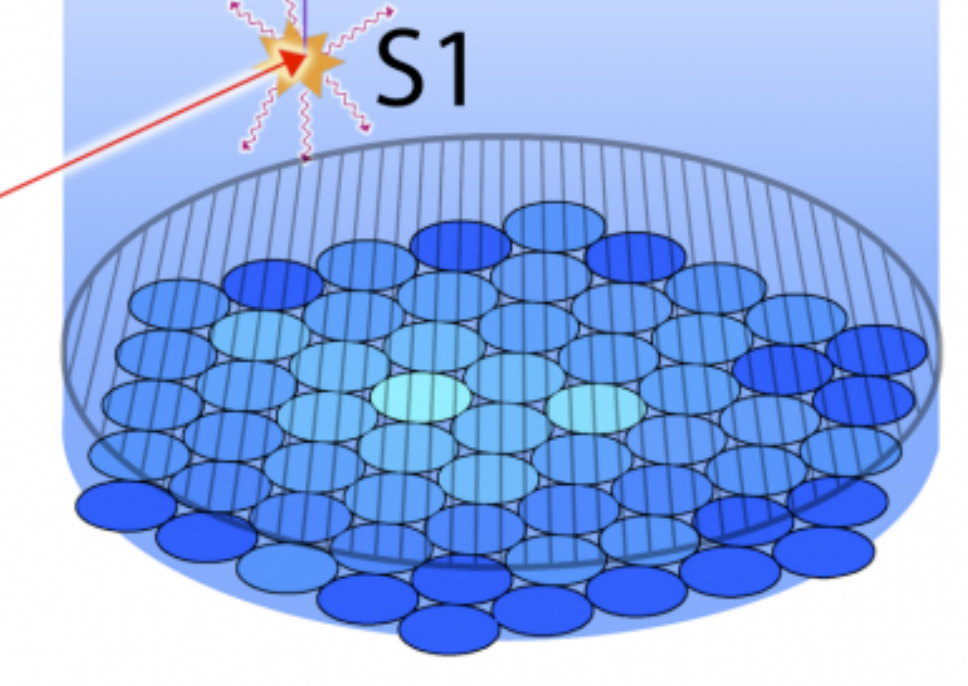
1 rejection of electron recoil events 


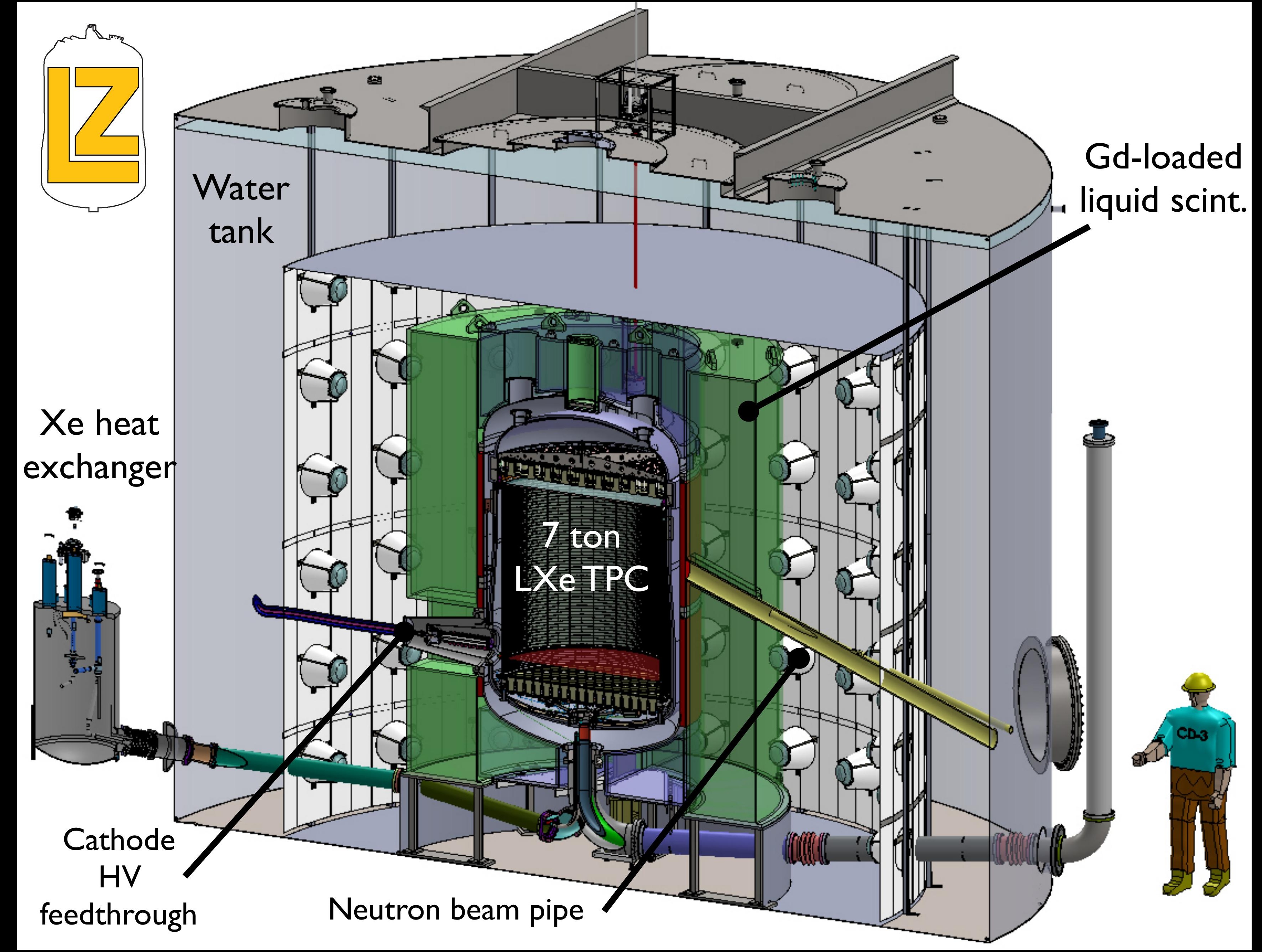




\section{LUX-Zeplin (LZ)}

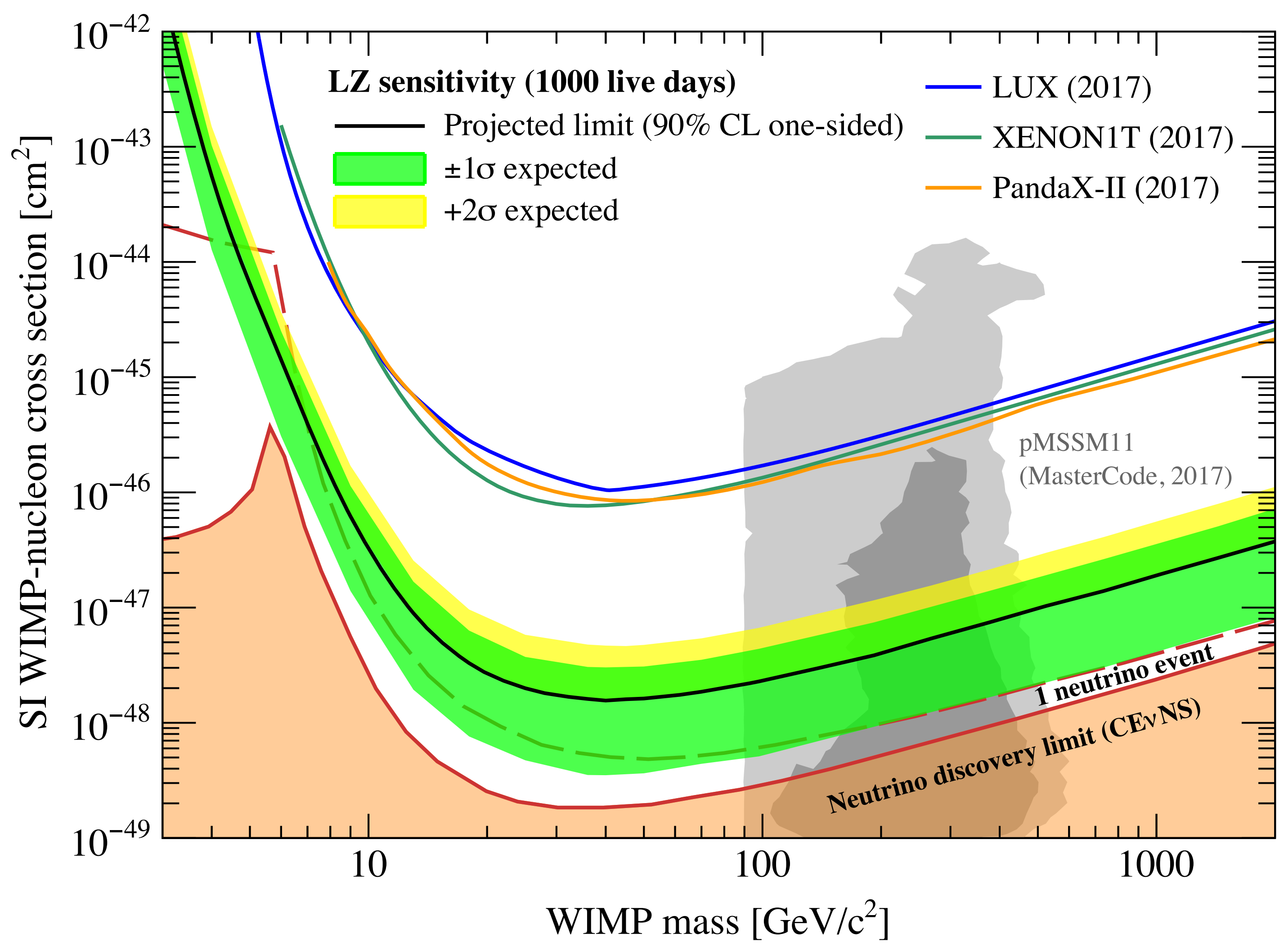




\section{Can we add He or H2 to LXe?}

- Dissolve small quantities of $\mathrm{He} / \mathrm{H} 2$ in liquid xenon

- Extend the reach of a detector like LZ (or XENONnT or PandaX, etc)

- Add new targets to field of direct detection

- No existing experiments using either

- Talk on HeRALD by H. Pinckney next

- NEWS-G gas detector in Canada another contender

- Capitalize on investment in large detectors by adding flexibility 


\section{Dissolving $\mathrm{He} / \mathrm{H}$ in $\mathrm{LXe}$ ?}

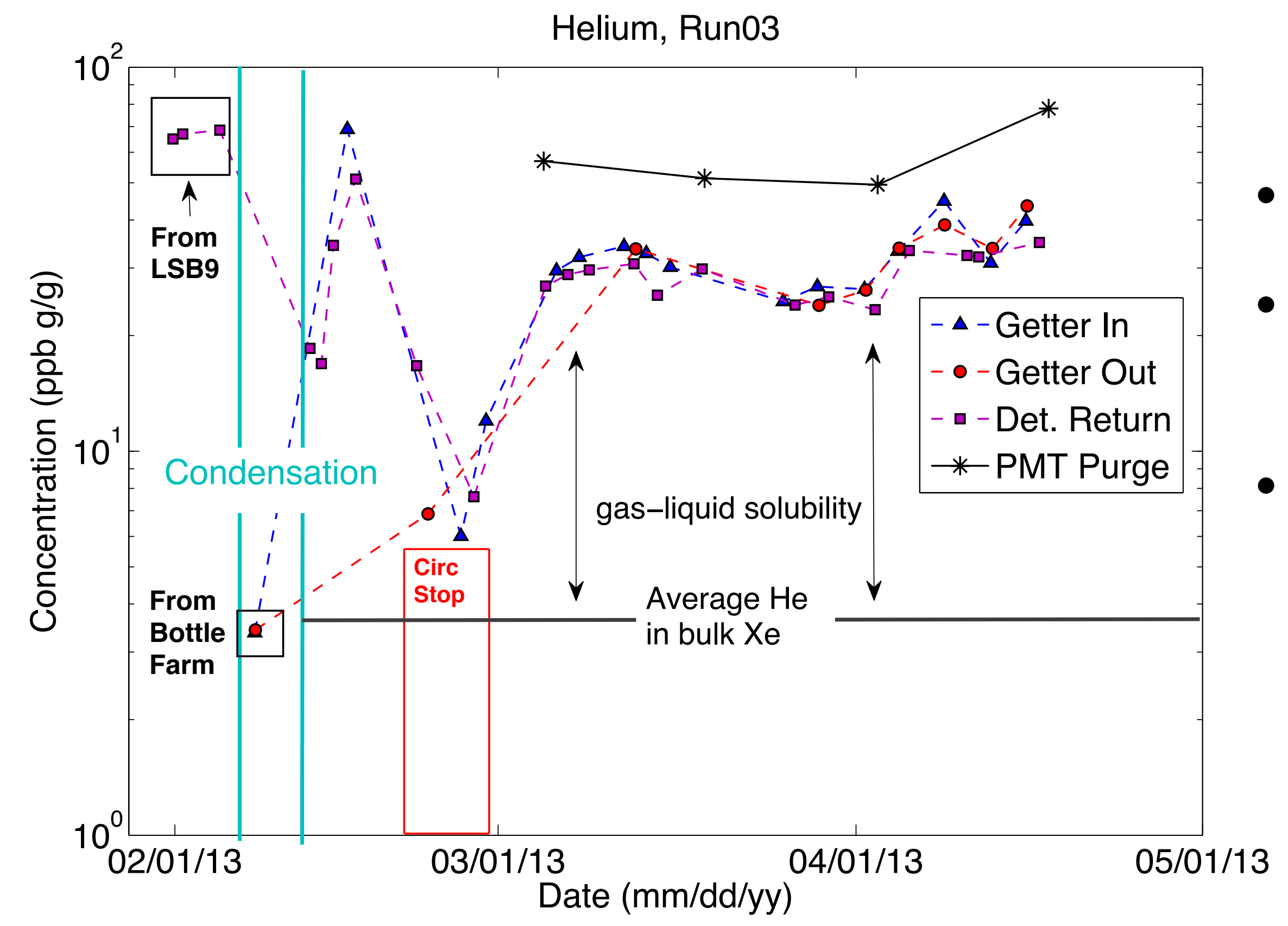

- LUX fill data

- Some residual He in the source bottles

- Data imply 3e-3 mass fraction for 1 atm partial pressure 


\section{Dissolving $\mathrm{He} / \mathrm{H}$ in $\mathrm{LXe}$ ?}

${ }^{4} \mathrm{He} /{ }^{129} \mathrm{Xe}$ Normalized to Gas Phase

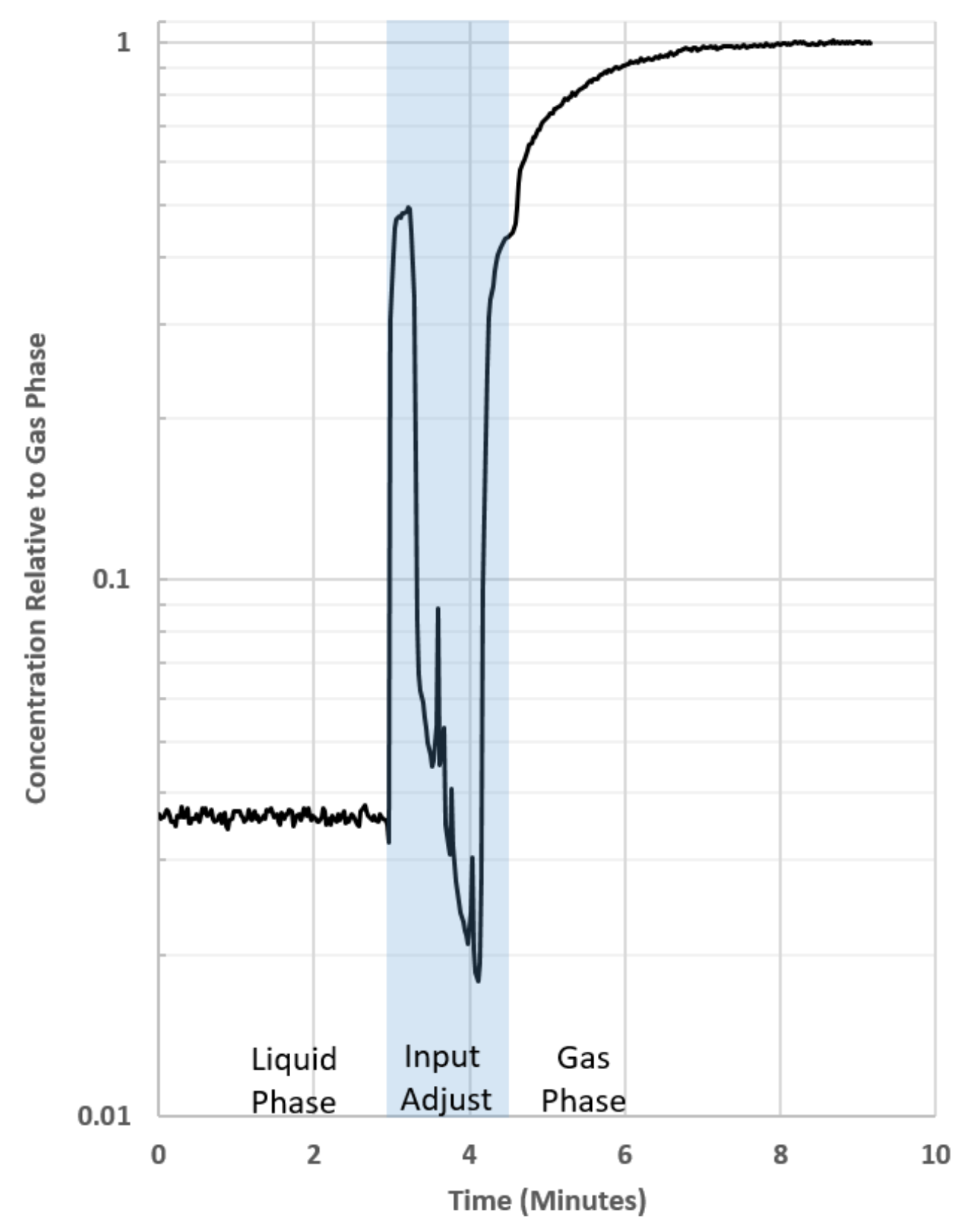

- He fraction confirmed in preliminary test at Fermilab

- Achieved 0.1\% He in LXe by mass on first attempt at 1 bar of partial pressure

- No data for $\mathrm{H}_{2}$ in xenon, but scaling by argon data, 25\% better than $\mathrm{He}$

$0.037 \mathrm{~mol} \mathrm{He} / \mathrm{mol} \mathrm{Xe} x$

$\mathrm{M}_{\mathrm{He}} / \mathrm{M}_{\mathrm{Xe}} \sim 0.1 \%$ 


\section{Backgrounds}

- The longest known radioisotope of $\mathrm{He}\left({ }^{6} \mathrm{He}\right)$ decays in $<1 \mathrm{~s}$

- No new backgrounds introduced (tritium?)
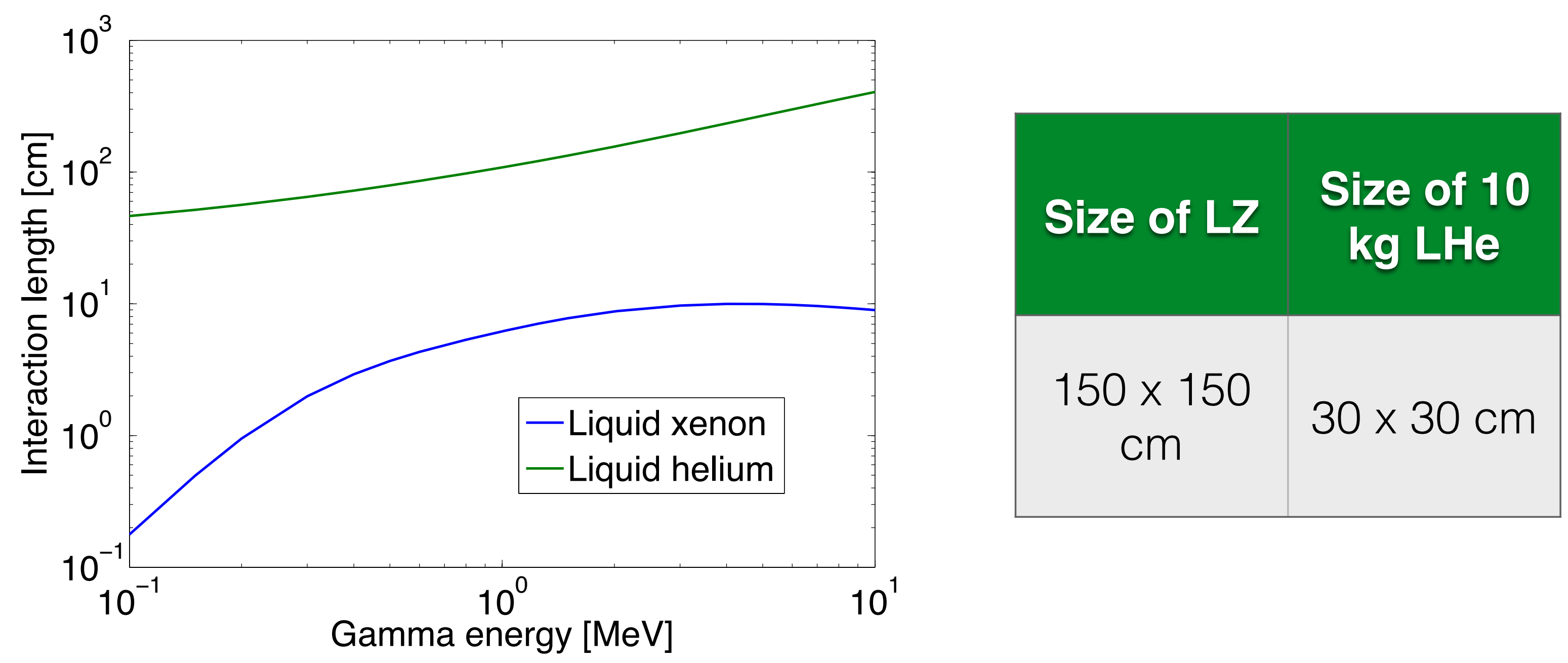

- Self shielding is not effective in $\mathrm{He} / \mathrm{H}$-only detector 


\section{Signal detection}

- Helium or Hydrogen recoils will interact with xenon atoms and electrons

- Excitations will be xenon excitations

- Alpha particles for example

- Keep same photon detection schemal

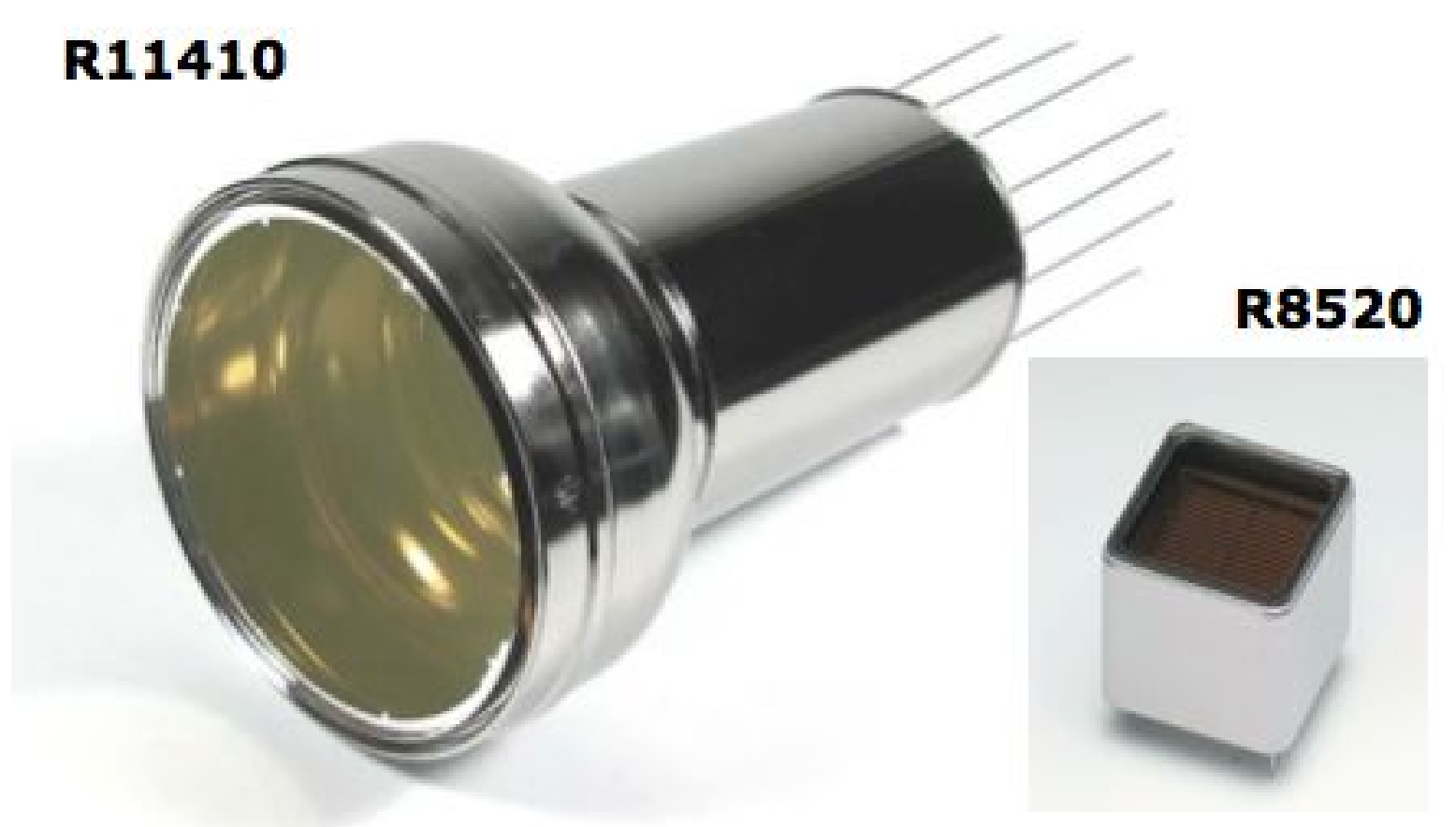




\section{Xenon microphysics}

- Xenon recoils in LXe lose a lot of energy to heat (Lindhard factor)

- Less than $20 \%$ of a $\sim<7 \mathrm{keV}$ recoil goes into detectable signal

- The rest goes into nuclear collisions that lead to heat

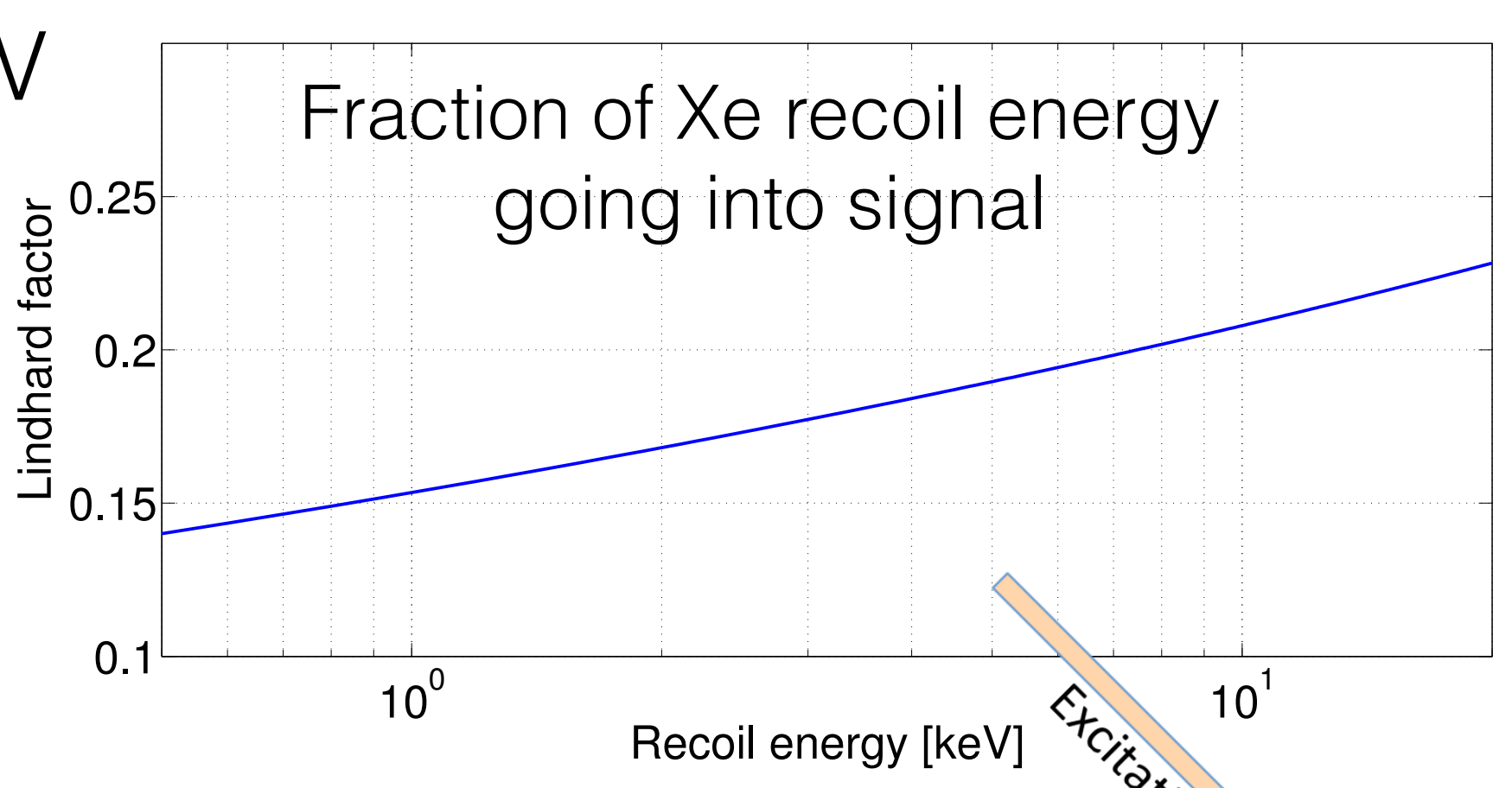

- Light nuclei - fewer strong nuclear collisions

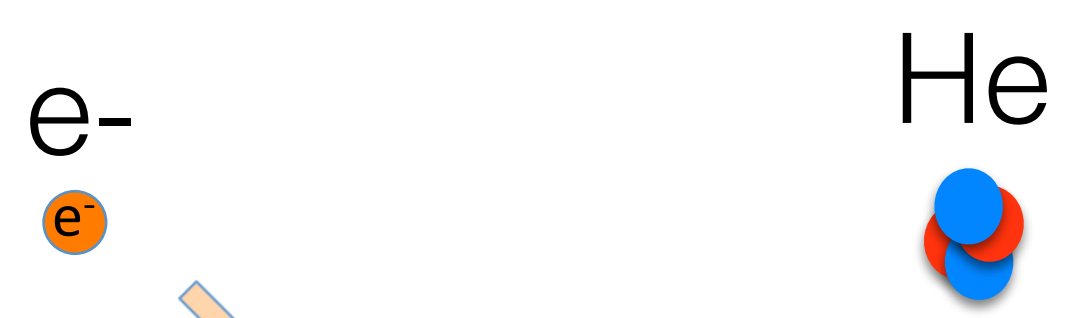




\section{Modeling He recoils in LXe (v1)}

- Stopping and Range of lons in Matter (SRIM)

- Calculate the energy lost to nuclear (heat) and electronic (signal) stopping

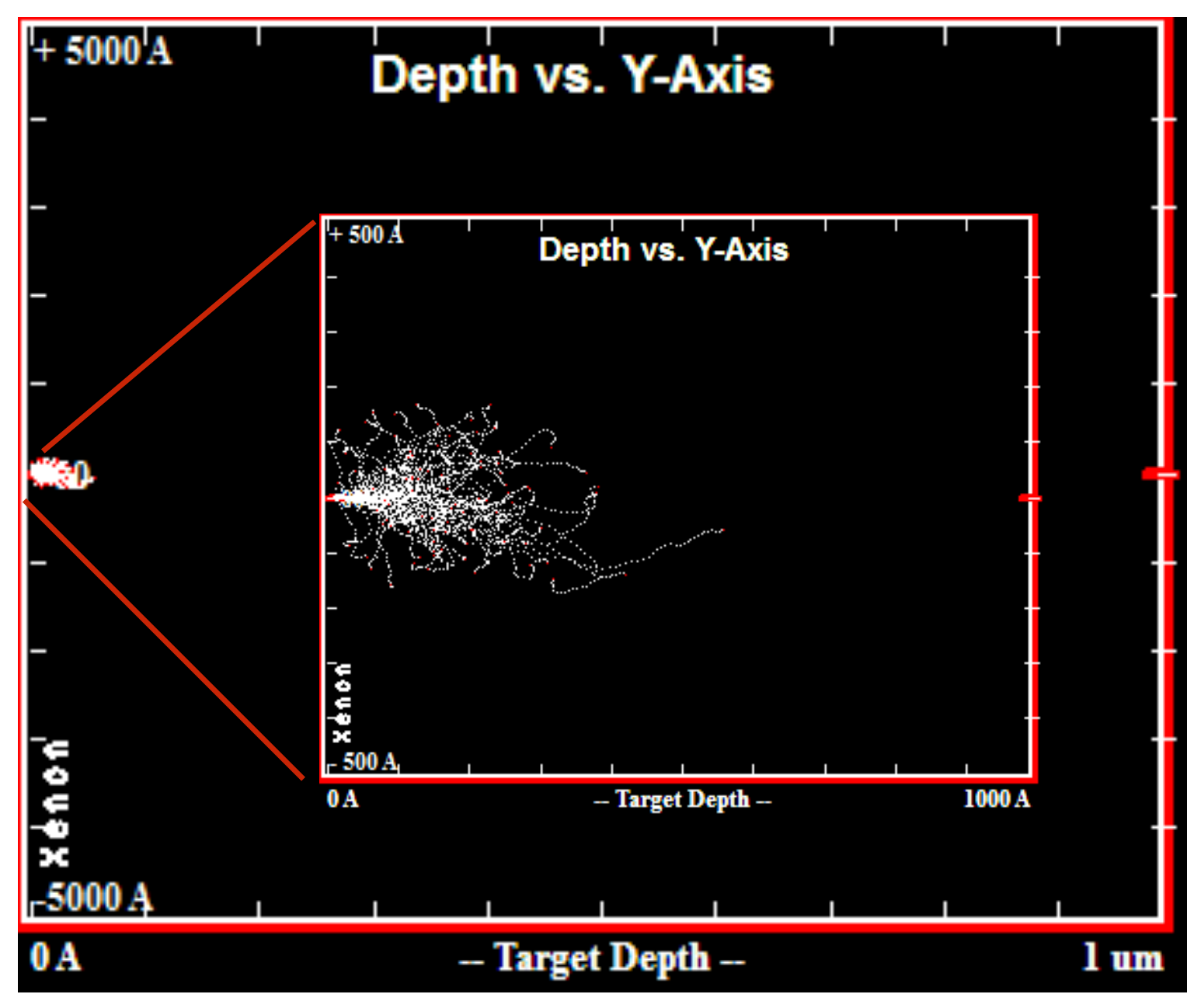

$10 \mathrm{keV} X e$ in $L X e$

$\sim 100$ A ranges

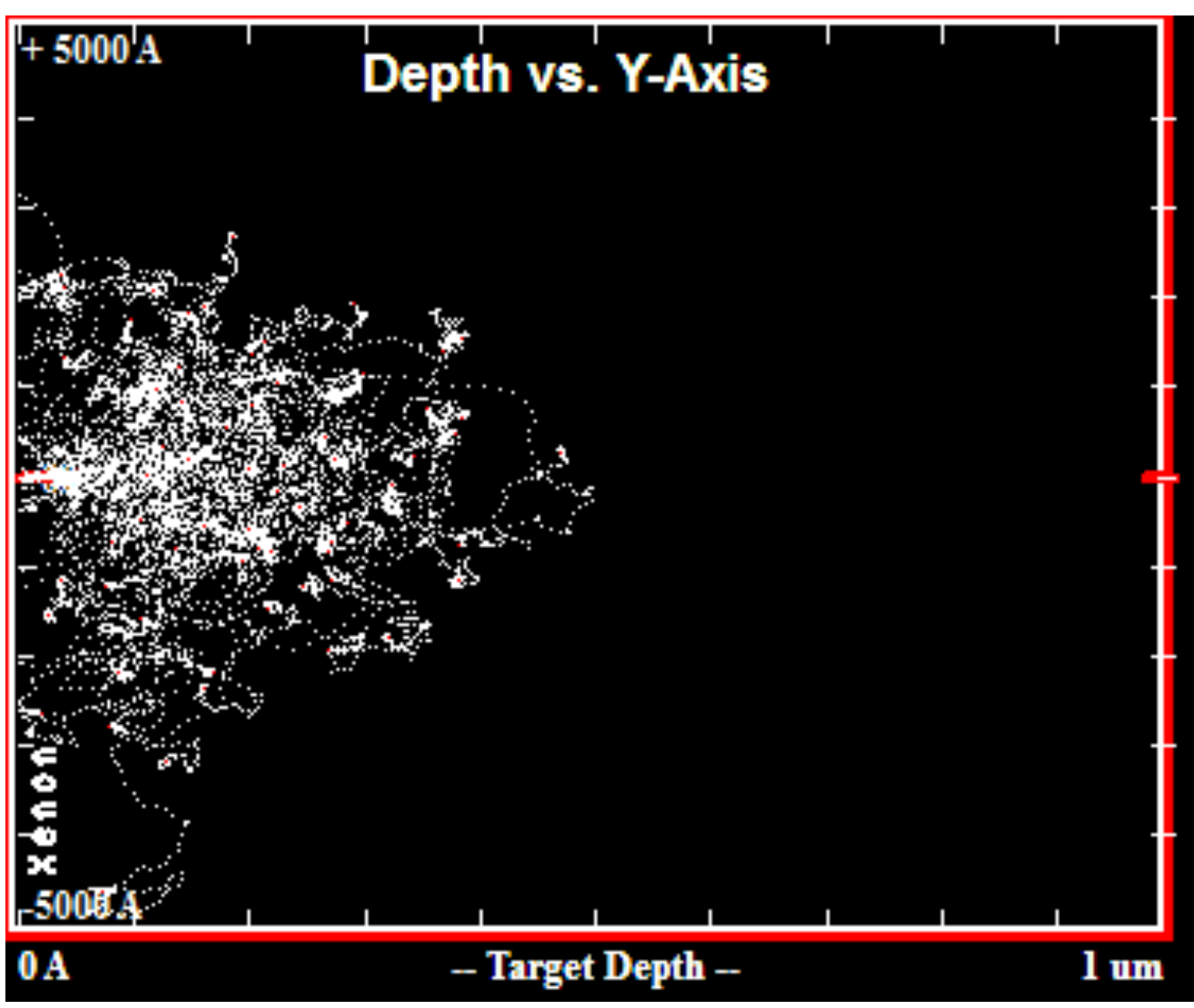

$10 \mathrm{keV} \mathrm{He}$ in LXe $\sim 1000$ A ranges 


\section{Modeling He recoils in LXe (v1)}

- Stopping and Range of lons in Matter (SRIM)

- Calculate the energy lost to nuclear (heat) and electronic (signal) stopping

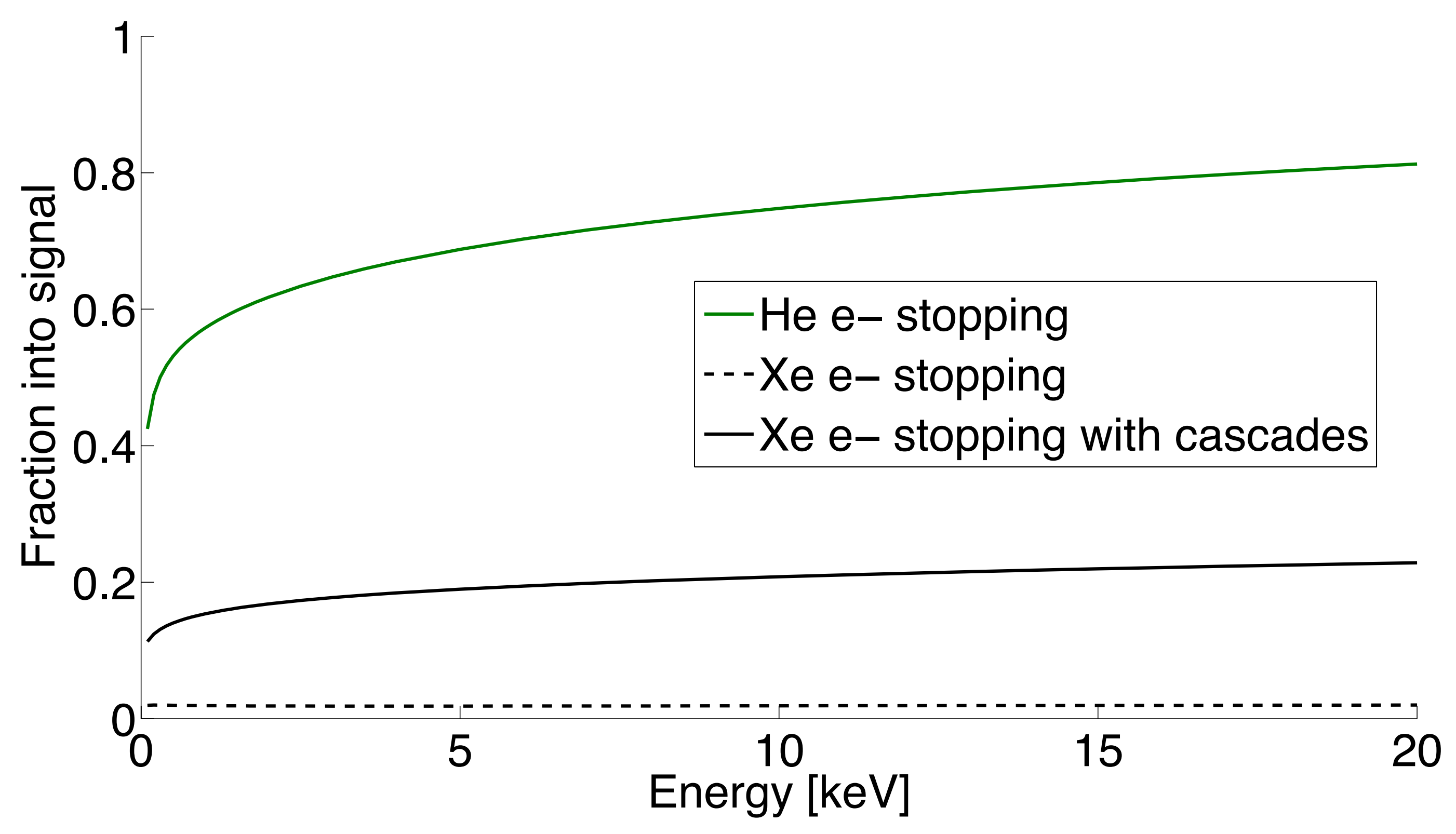




\section{Modeling He recoils in LXe (v2)}

- Noble Element Simulation Technique (NESTV2)

- Data driven model for signal processes in LXe, including alpha data from LUX and test chambers

- High energies, but at least it's real He nuclei in LXe

Light Yield vs. Recoil Energy from $\alpha$-particles

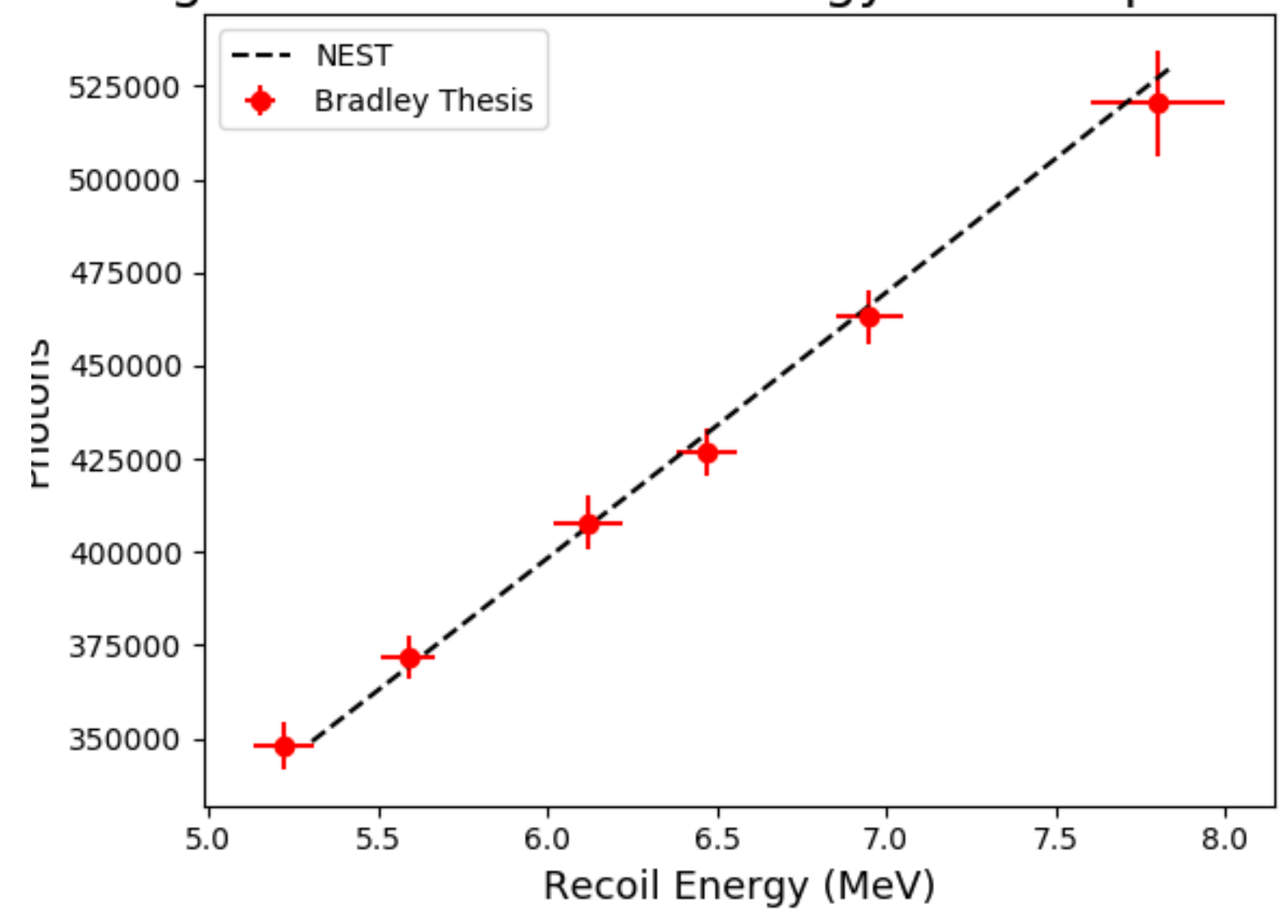

${ }^{210}$ Po Charge Yields from $\alpha$-particles

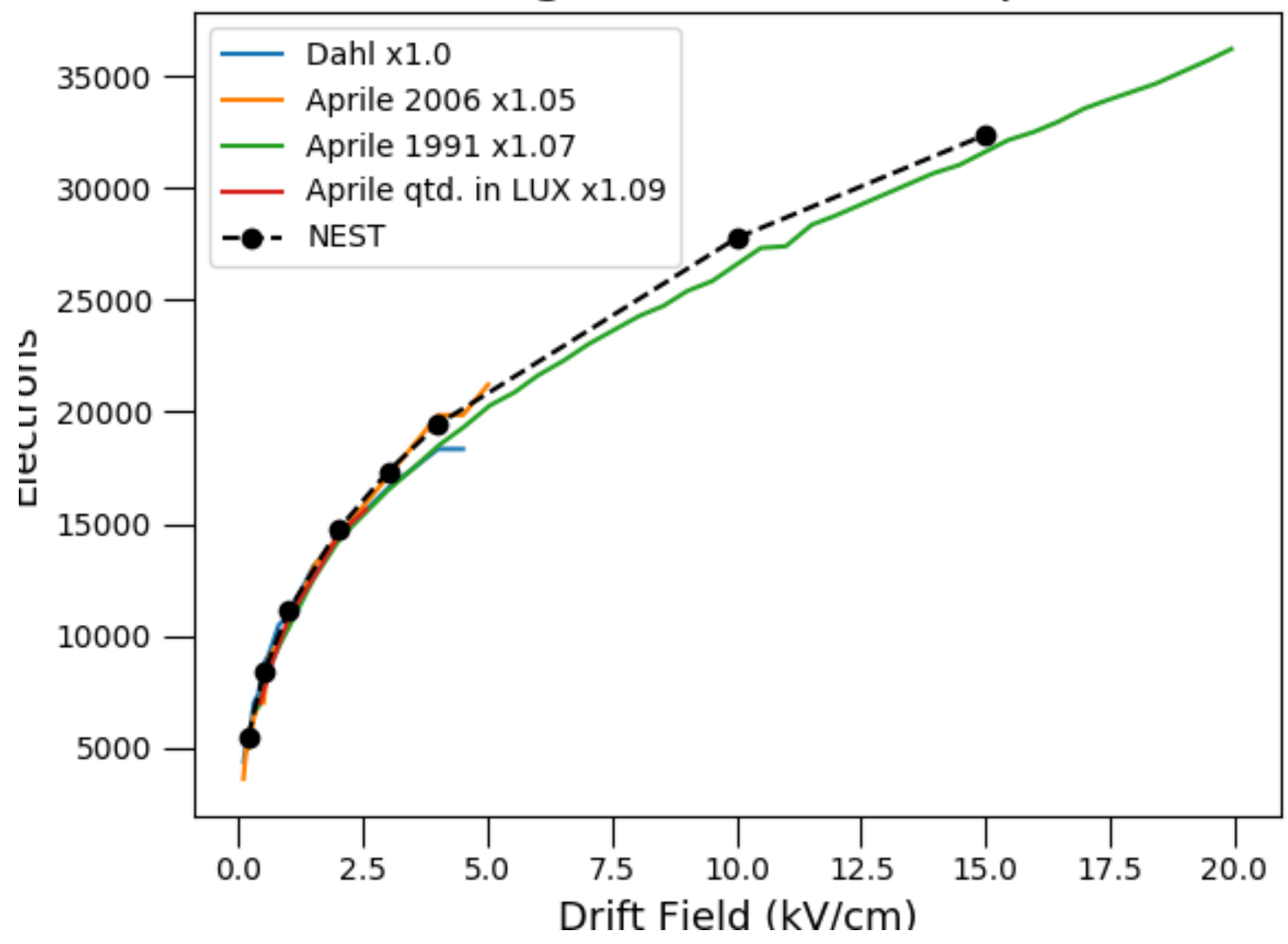


Modeling He recoils in LXe $(v 1+2)$

Total quanta $=$ hv $+e$

Factor > 3 more signal from $\mathrm{He}$

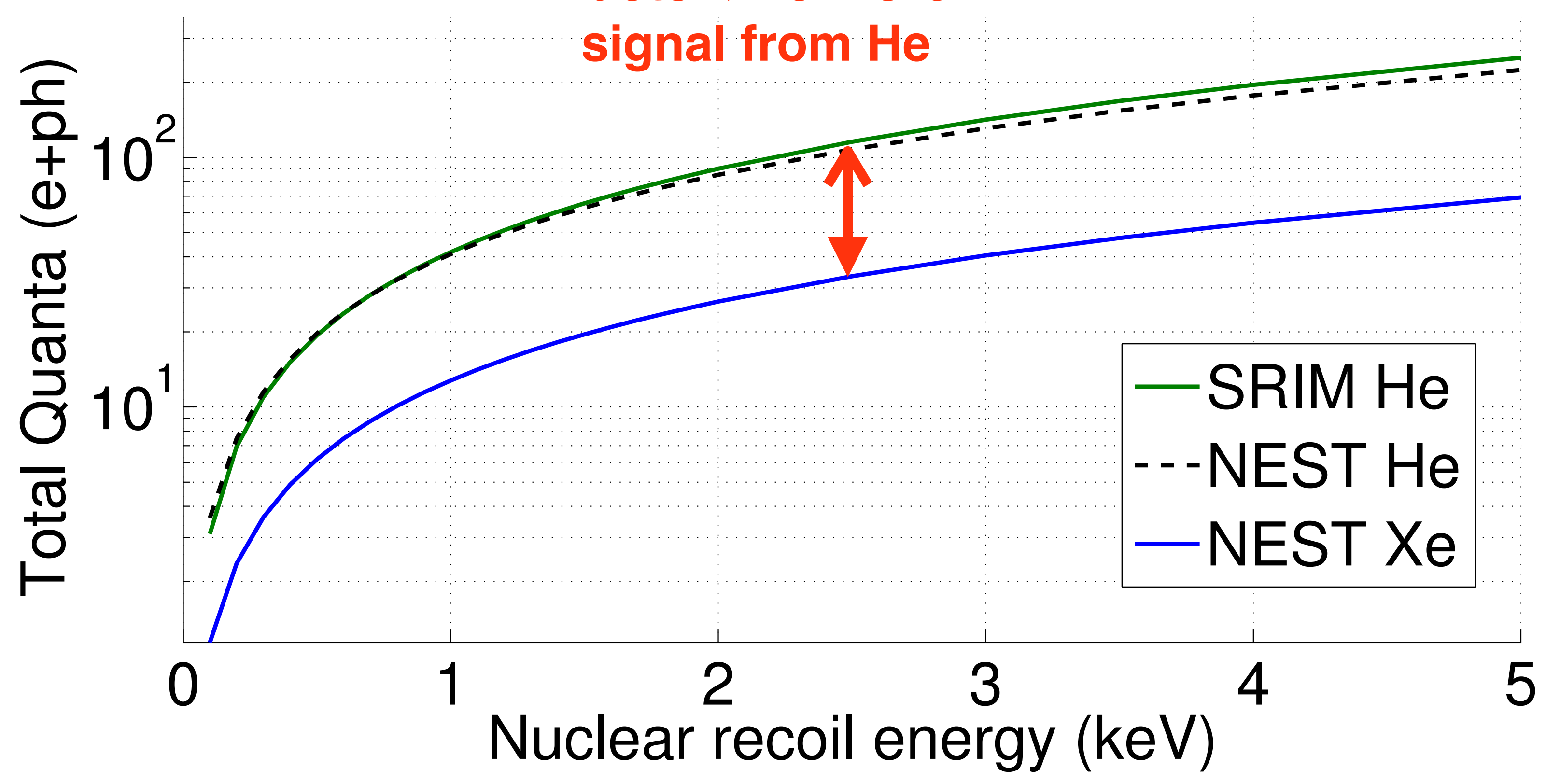




\section{Modeling $\mathrm{H}$ recoils in LXe (SRIM)}

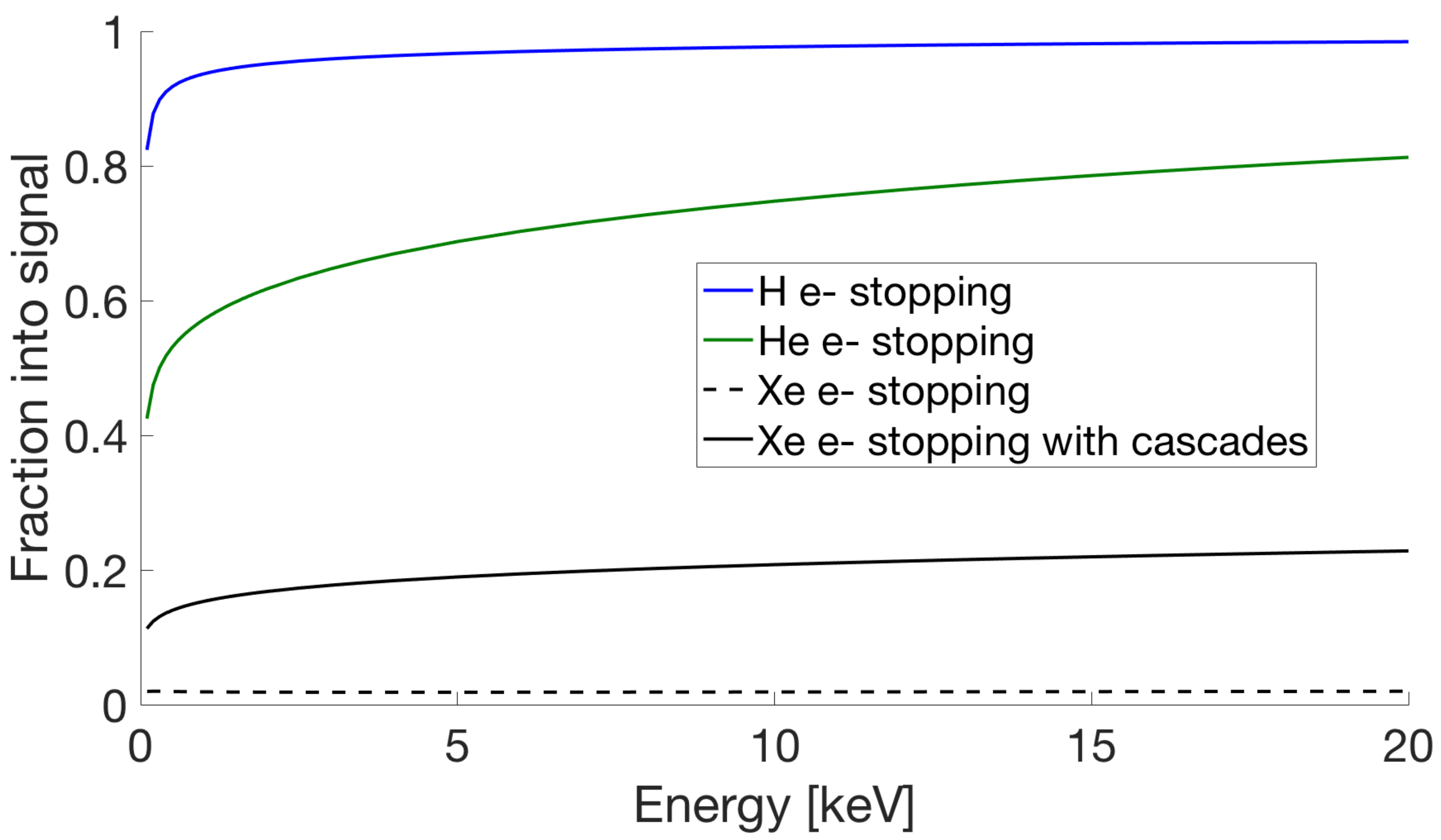




\section{A key question}

- What happens to S2/S1 partitioning?

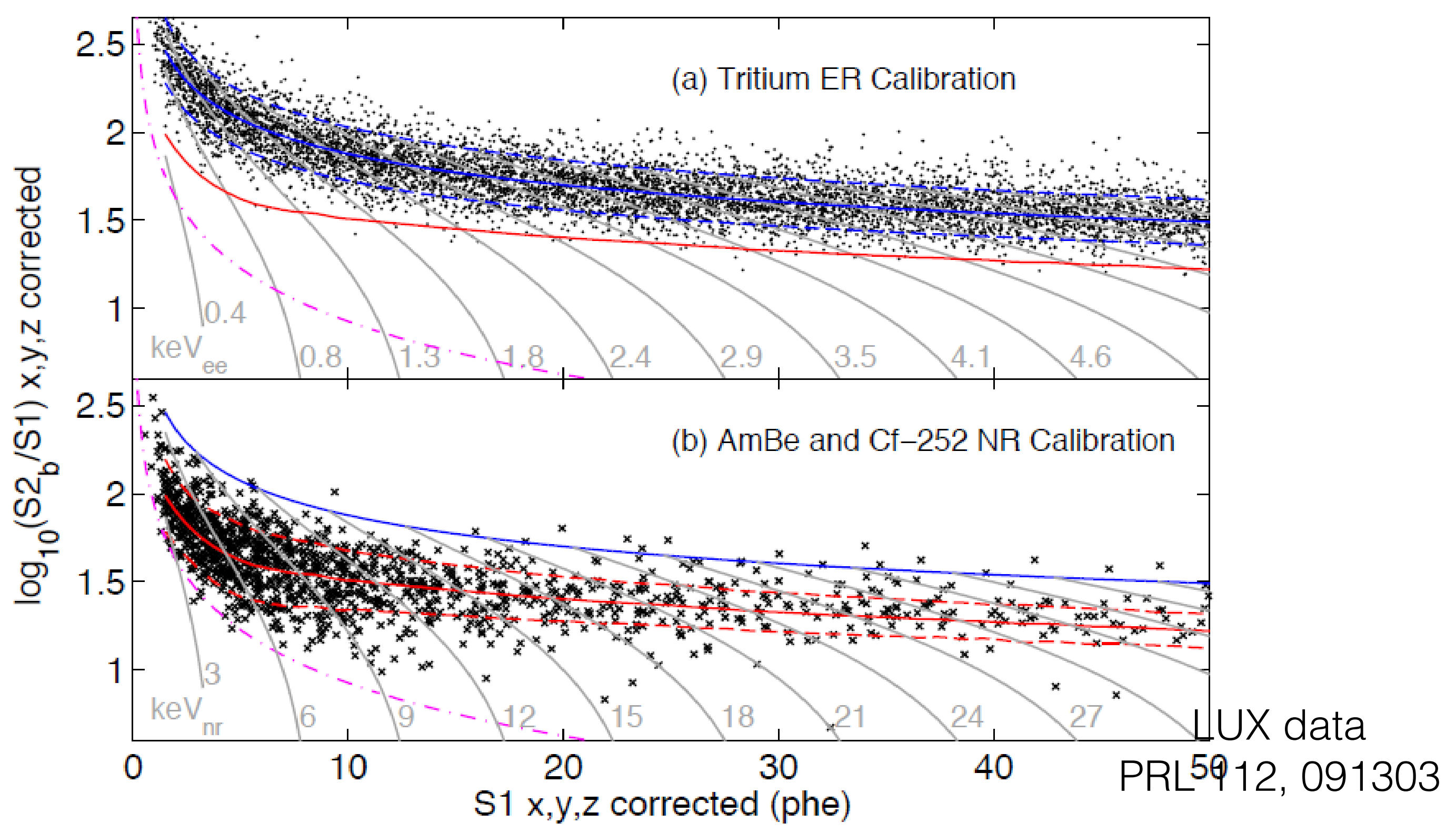




\section{Xenon microphysics}

- What happens to S2/S1 partitioning?

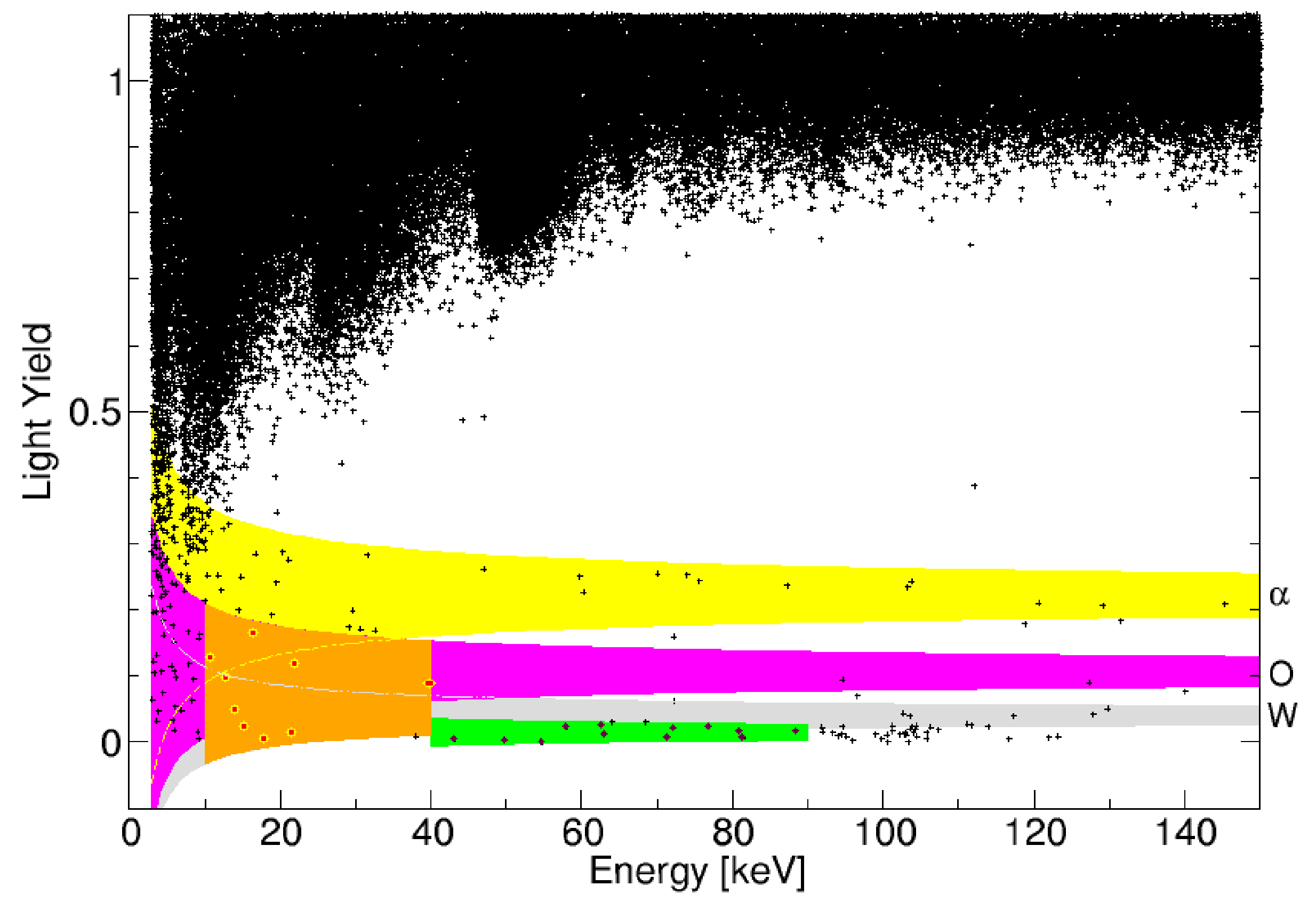

CRESST data in scintillating bolometers

NB: Different microphysical process (heat v. electronic) 


\section{What does it look like in LZ?}

- Put this all together into single model

- Use the LZ Geant4 detector and optical transport model

- See "Projected Sensitivity of LZ" (1802.06039)

- For S1/S2 analysis, threshold is determined by S1

- Partitioning into photons and electrons matters

- Run extreme cases for He - NR-like and ER-like

- Used SRIM for H - looks similar but slightly better 


\section{Energy threshold}

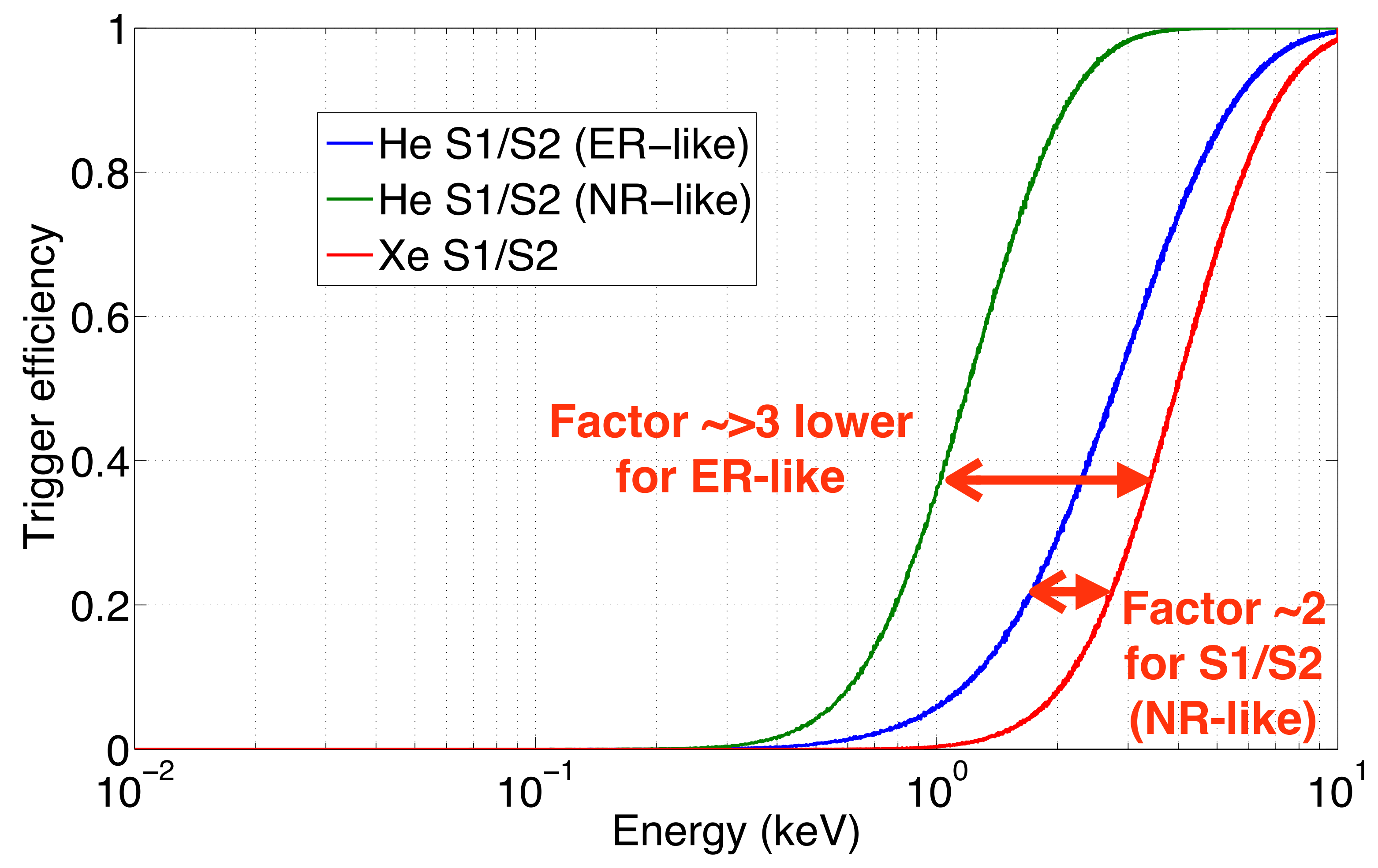




\section{S2-only analysis}

- Photon detection efficiency (S1) is about $10 \%$

- Electron detection efficiency is (we hope) about 100\%

- High gain on S2 channel (80 phd/e-)

- Enables much lower threshold if you look at "S2-only"

- Give up ER/NR discrimination

- Subject to single electron noise

- Still very powerful

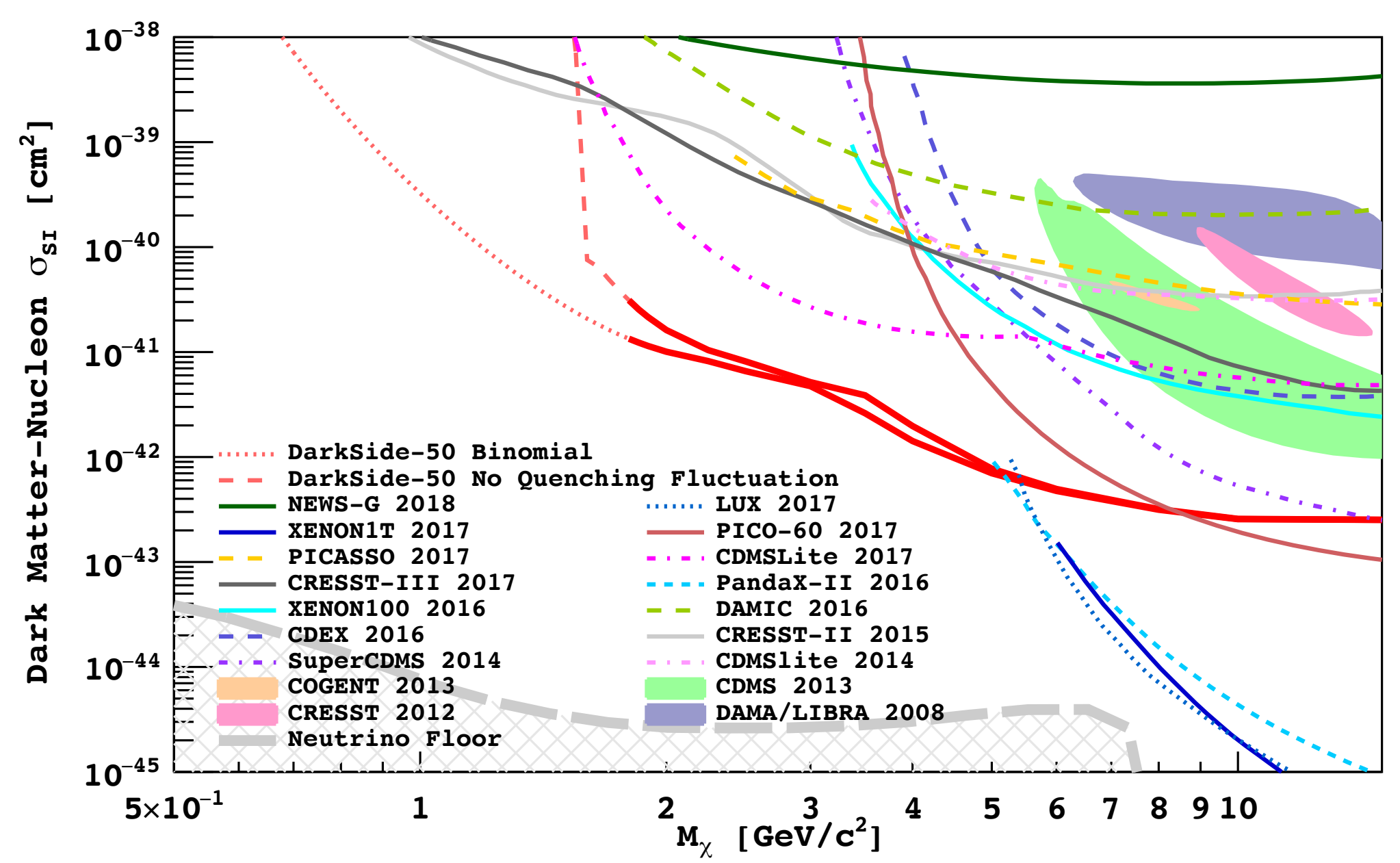




\section{Energy threshold}

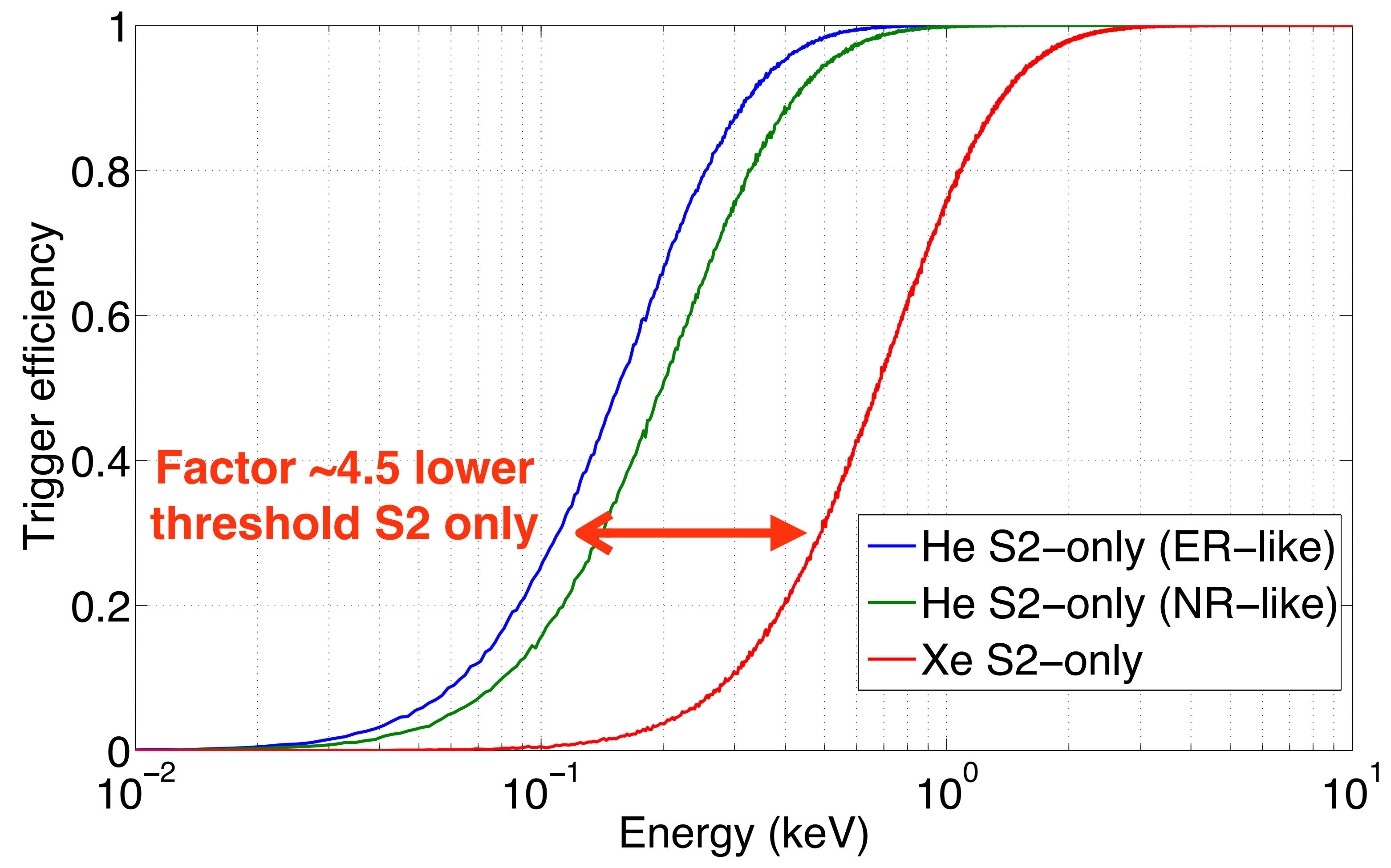

- 3 electron threshold assumed for S2 (>250 photons) 


\section{Making projections}

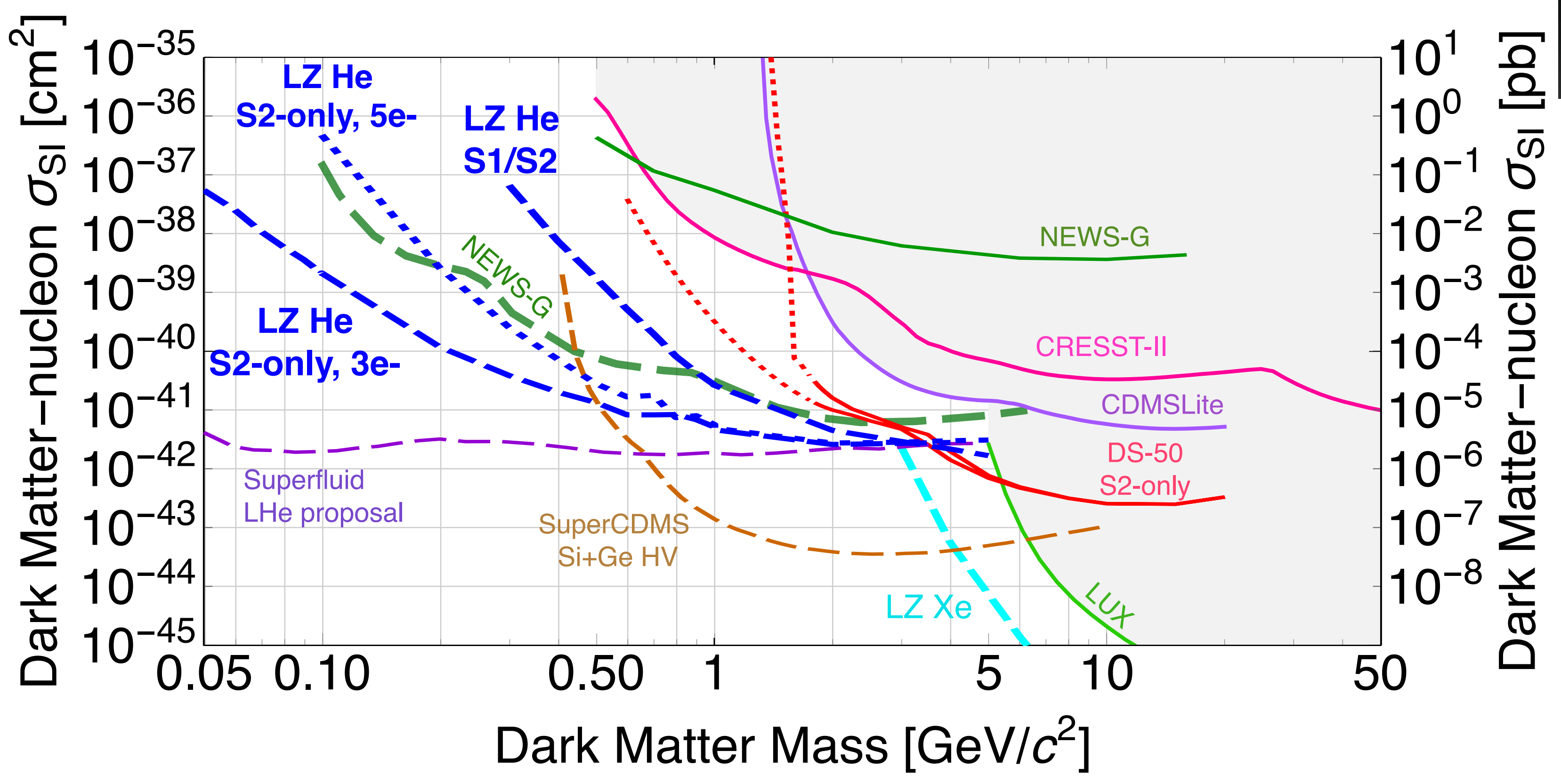

- 0.3\% loading (1 bar partial pressure) - 15 kg, 20 days for S2-only, 100 days for S1/S2

- Location of LZ Helium lines depends critically on assumed signal yield

- 225 events/day/pb with S2 only at $100 \mathrm{MeV}$ WIMP with this yield

- Dotted line is 5e- S2-only threshold 


\section{With Hydrogen}

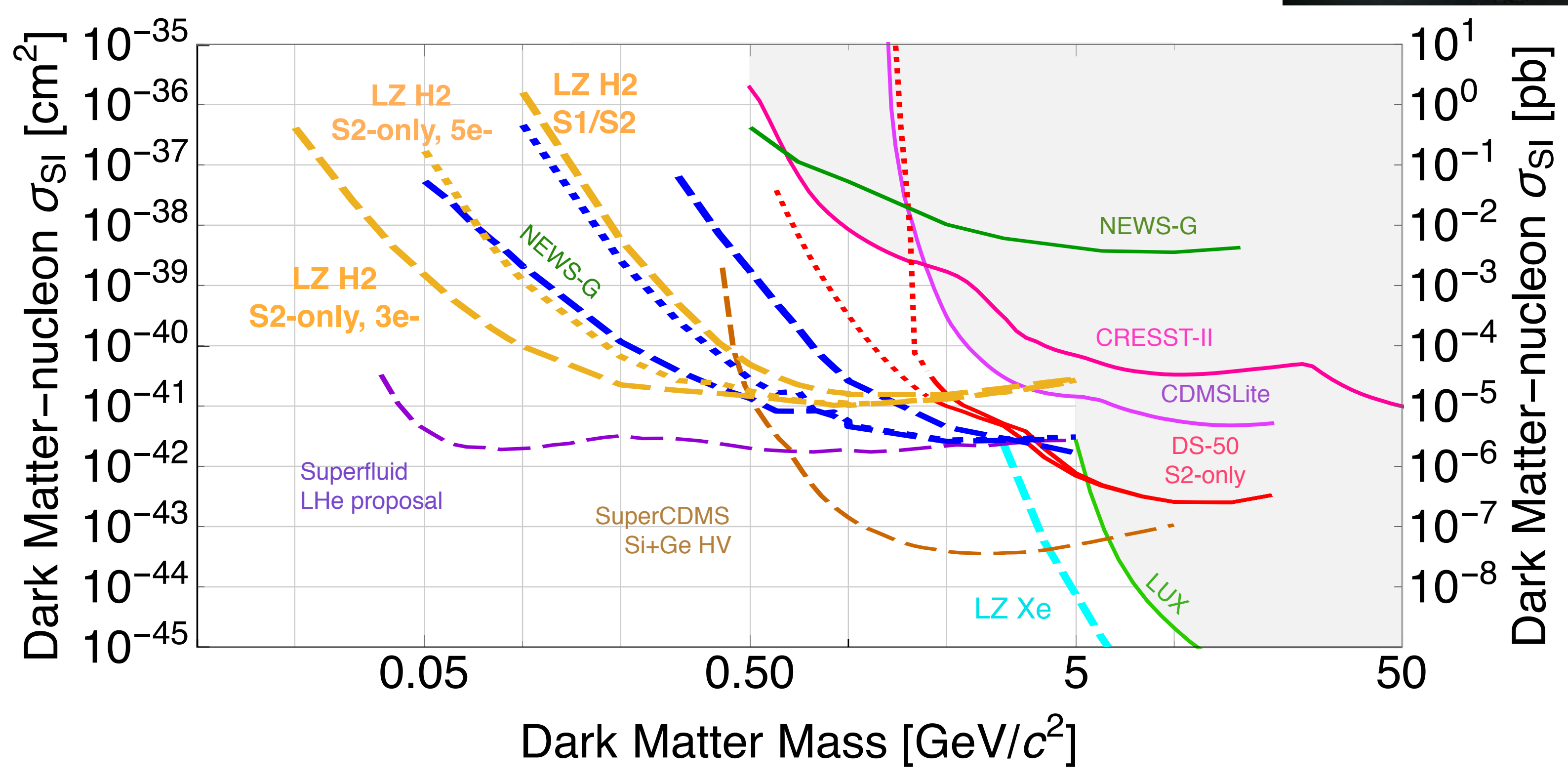

- Projection from calculating yields with SRIM + LZ detector model

- Definitely to be taken with grain of salt

• $0.0375 \%$ H2 (0.1 bar partial pressure), $1.9 \mathrm{~kg}, 500$ days 


\section{SD Hydrogen}
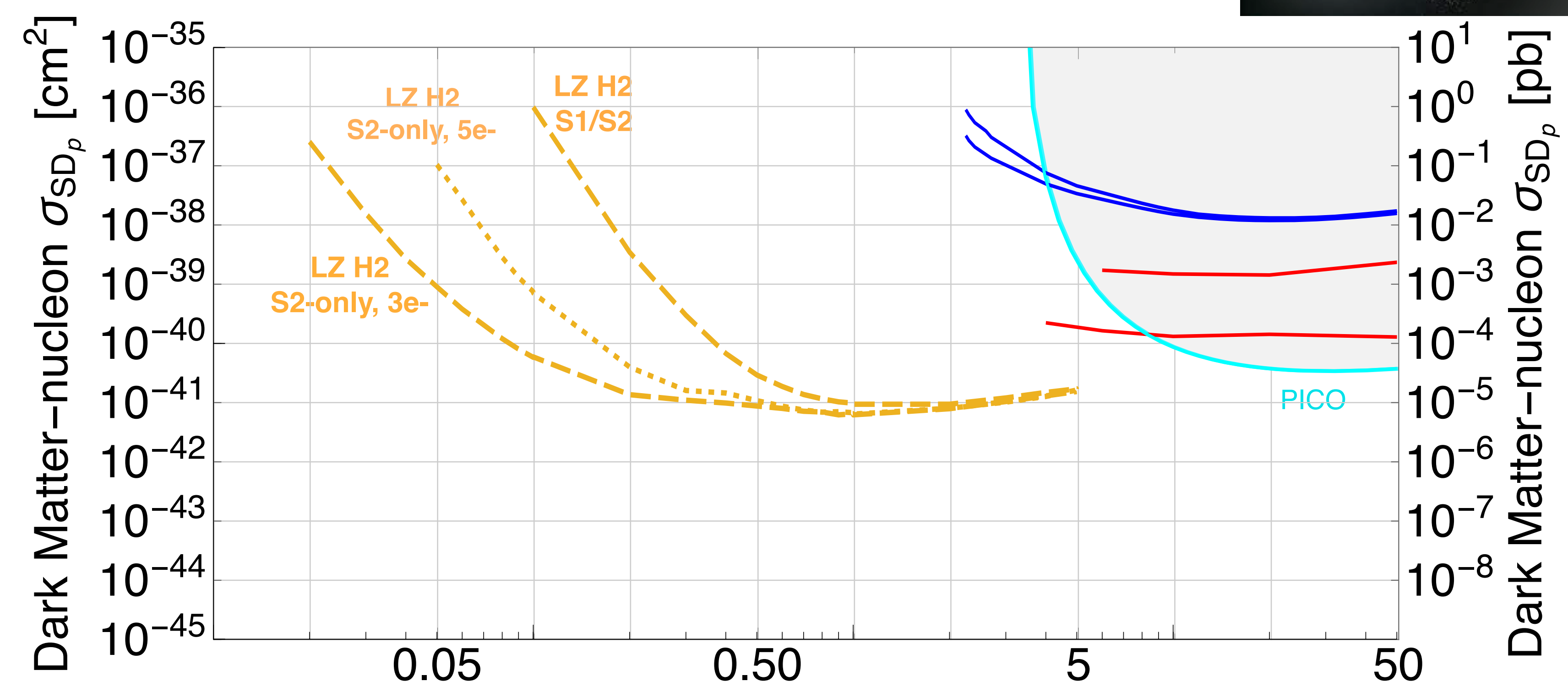

Dark Matter Mass $\left[\mathrm{GeV} / \mathrm{c}^{2}\right]$

- Projection from calculating yields with SRIM + LZ detector model

- Definitely to be taken with grain of salt

- $0.0375 \%$ H2 (0.1 partial pressure), 1.9 kg, 500 days 


\section{What do I worry about}

- Helium gas and PMTs are not a good mix

- Diffusion exponentially suppressed by temperature (Arrhenius relationship)

- Calculation suggests 500 days at 1 bar/ $165 \mathrm{~K}$ before tube becomes inoperable

Example for ET9226 PMT

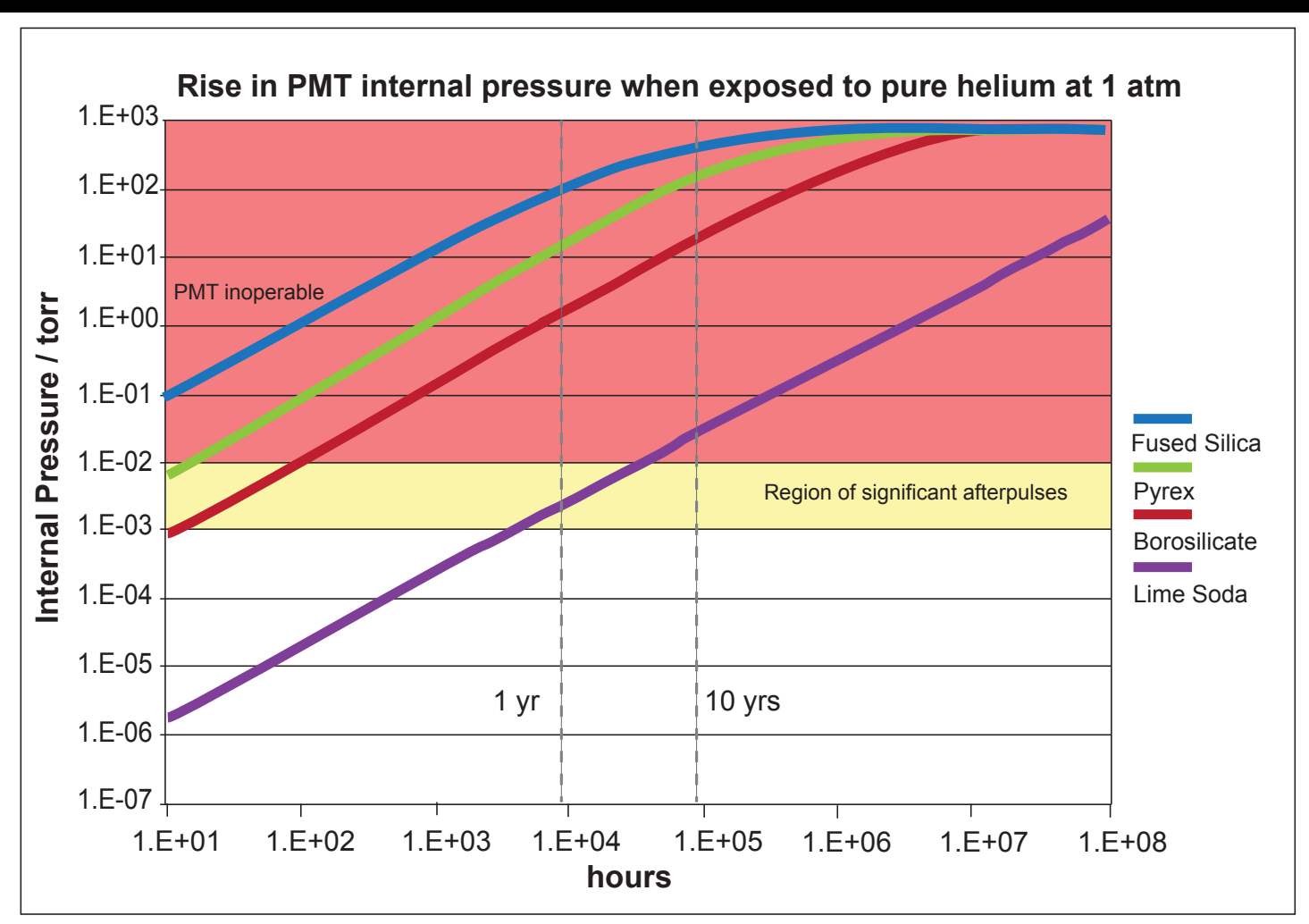

- Exquisitely sensitive to temperature,

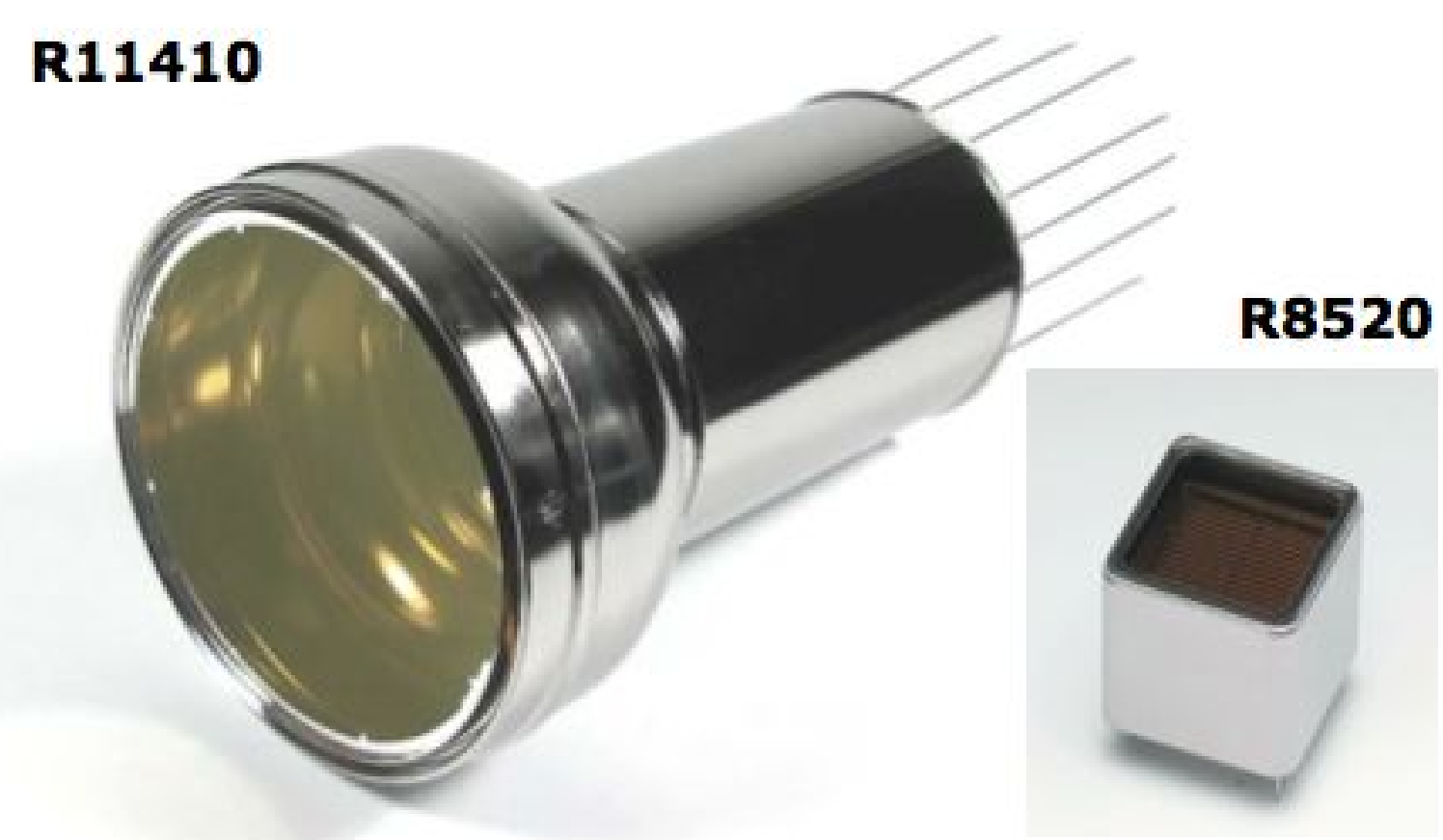
and that's pretty tight...

- Needs to be tested

- Could use SiPMs... 


\section{What do I worry about $(\mathrm{H})$}

- PMT diffusion is suppressed

- Hydrogen is flammable in mine environment

- Purification - getter will take out the $\mathrm{H} 2$

- Suppression of S2 production

- Molecular modes can slow down electrons

- Could recover with increased voltage 


\section{What do I worry about}

- This is still fairly speculative

- Henry's coefficients not comprehensively measured

- Temperature dependence, diffusion, etc?

- Signal yields depend on modeling and MeV scale data

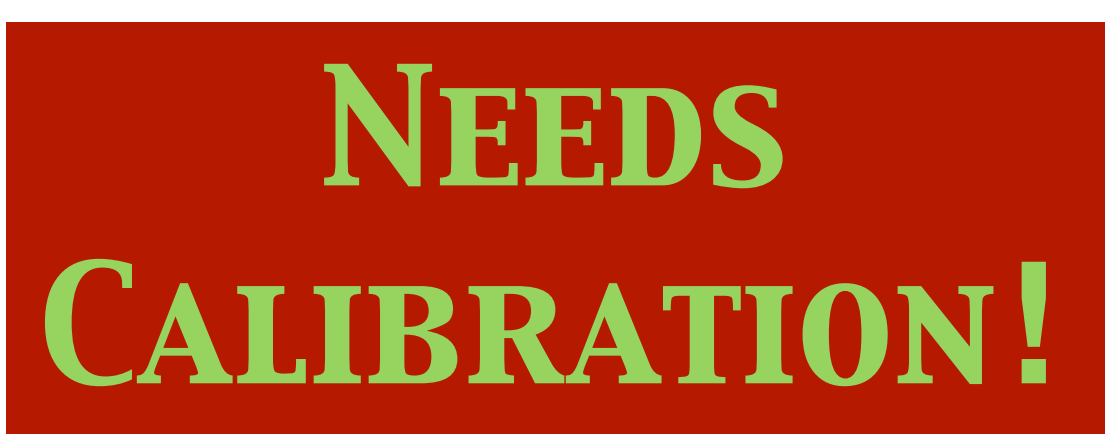

- Monoenergetic neutron scattering experiment is where I would start 


\section{What do I worry about}

- Cryogenics - what does the presence of the non-condensible gas do to our cryogenics

- Bubble $\mathrm{He} / \mathrm{H} 2$ through the bottom of the cryostat?

- Phase separated at weir drain (in LZ design)?

- Should be distilled out fairly efficiently

- Introduction and mixing that worries me the most 


\section{$\mathrm{He} / \mathrm{H}$ doping in $\mathrm{LXe}$}

- Physically possible

- Keep low background level achieved in LXe TPC

- Same signal readout with LXe sensitive light detectors

- Increased signal yield from He recoils

- Lower energy thresholds for WIMP-He scattering

- Properties measurable using existing techniques

- Potential reach to well below $1 \mathrm{GeV}$ dark matter

- Depends on properties that need to be measured 


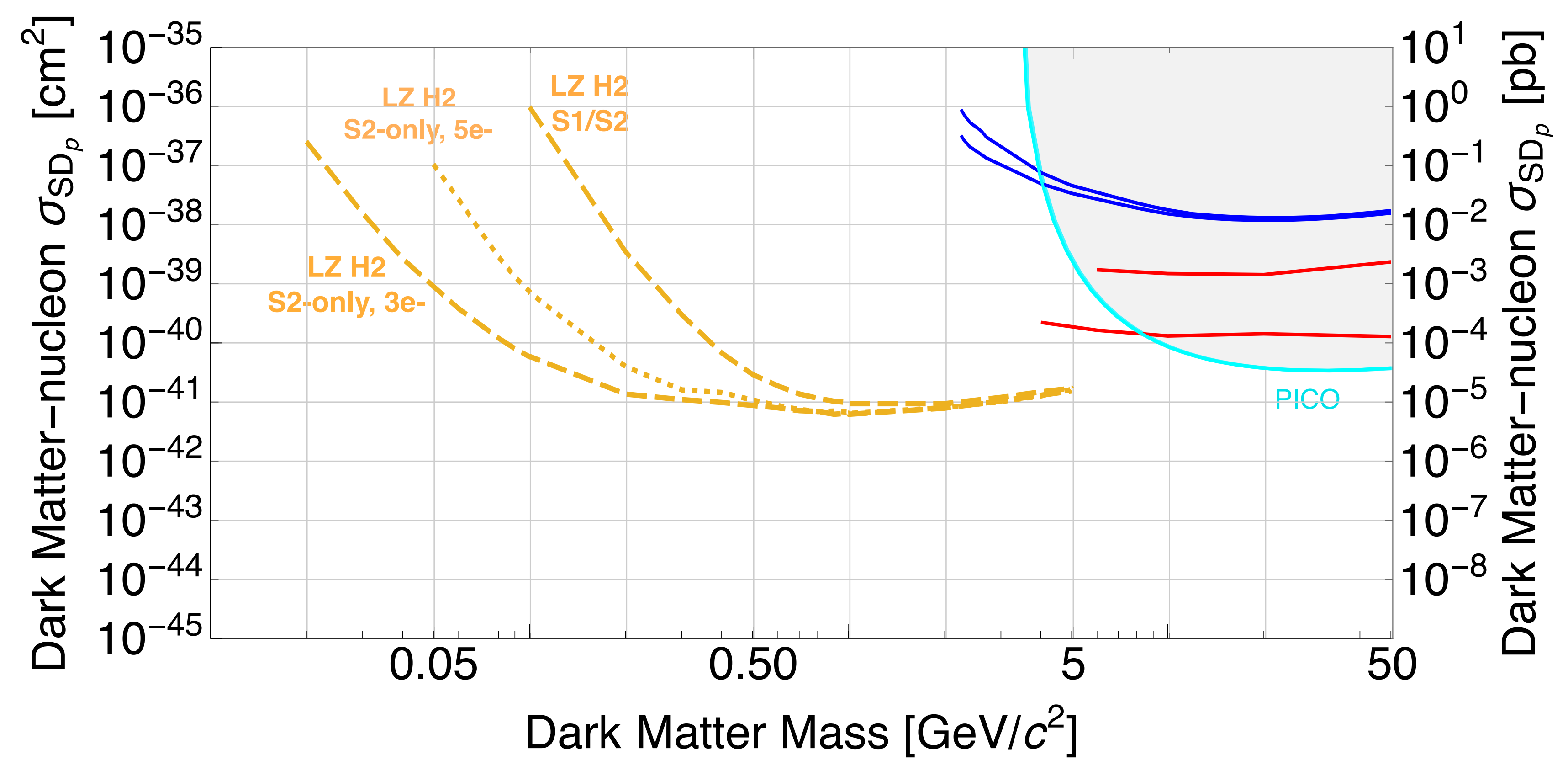




\section{Backup}




\section{Neutron scattering measurement}

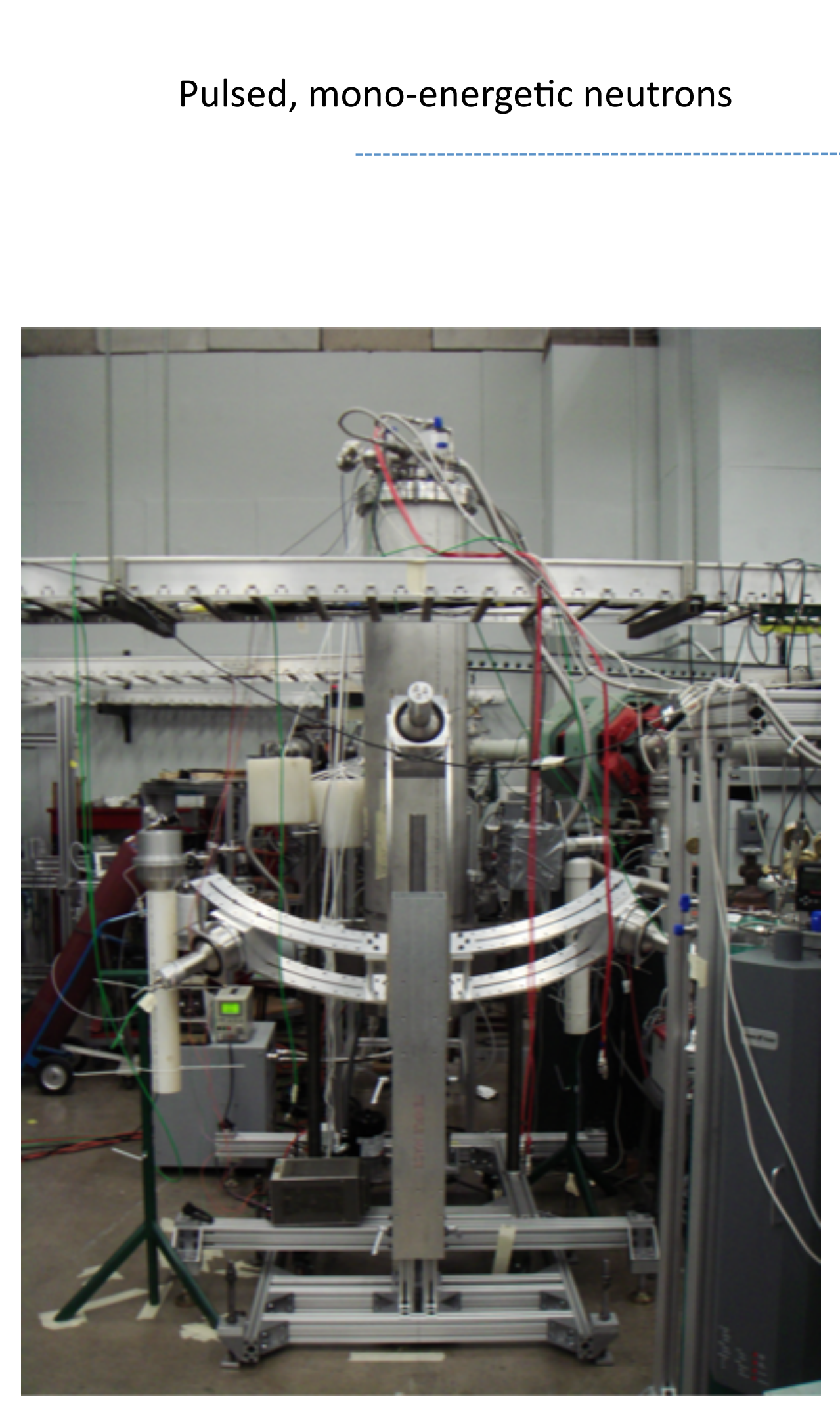

TPC

\section{(5)}

Scattering angle, $\Theta$

Neutron detector

- Pulsed, monoenergetic beam (at Notre Dame or elsewhere) to measure response of to nuclear recoils of known energy

- Tunable nuclear recoil energy by changing the neutron energy and the scattering angle

- Neutrons of $100 \mathrm{keV}-1.5 \mathrm{MeV}$

- Recoils of $\sim 1 \mathrm{keV}$ up to $50 \mathrm{keV}$

- Successful measurements in LAr (1406.4825, 1306.5675, SCENE) 


\section{Neutron scattering in SCENE}

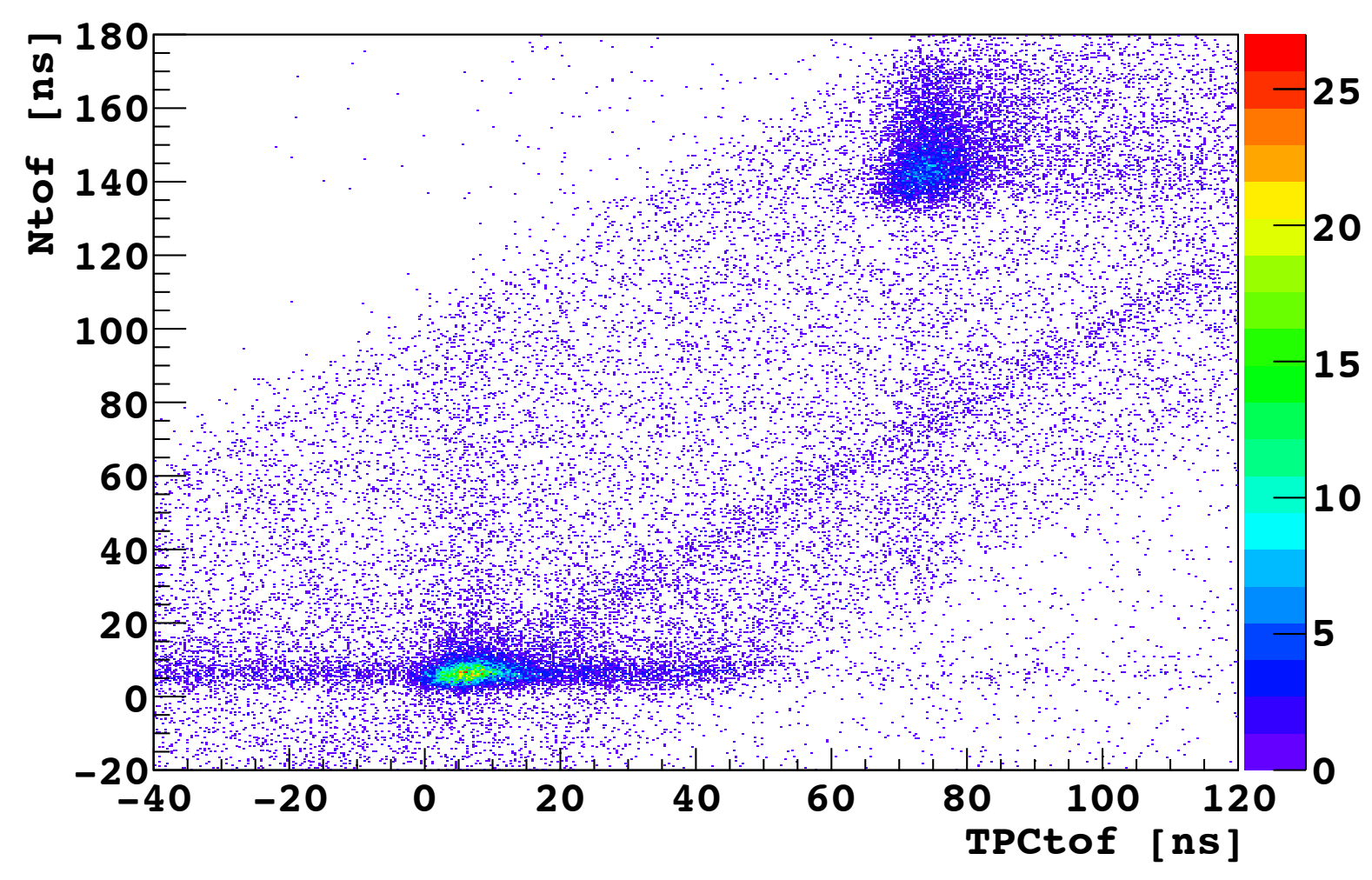

- Time of flight to measure the neutron timing

- Pulse shape discrimination(PSD) to select neutrons in the detectors

- Ntof - time between beam pulse and neutron detector

- TPCtof - time between beam pulse and LAr detector

- $f 90$ - PSD in LAr

- Npsd - PSD in neutron detector
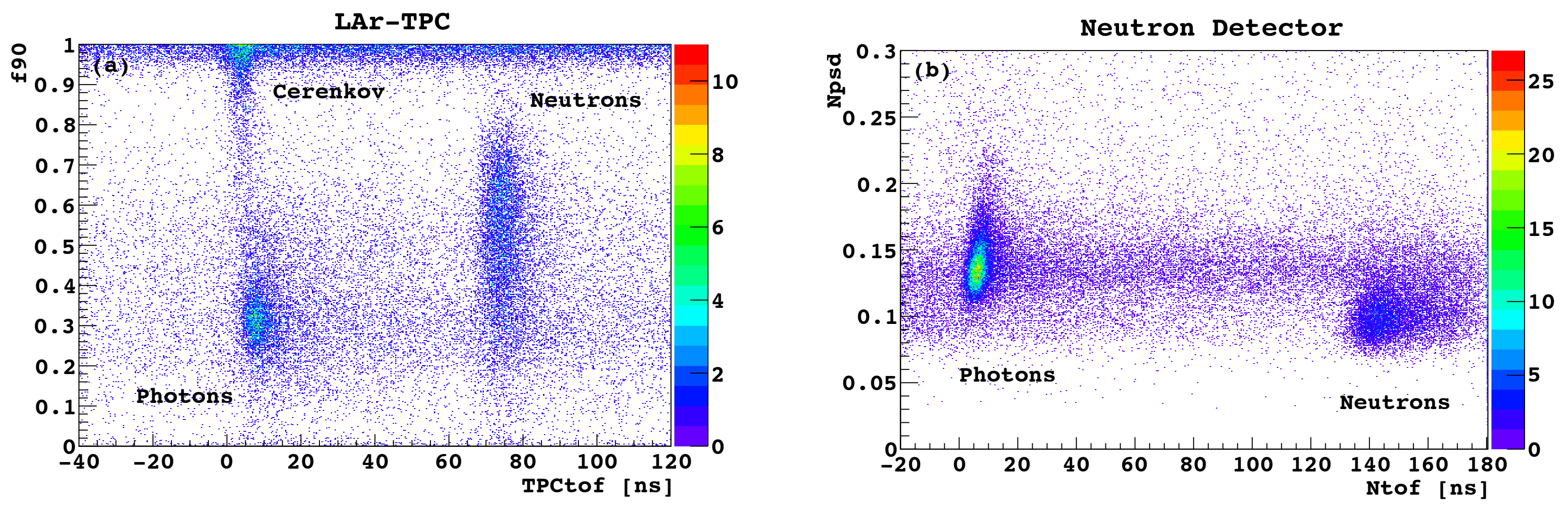


\section{Neutron scattering with $\mathrm{He}$ in $\mathrm{LXe}$}

- In a doping measurement, for a given scattering angle, He recoils have more energy

- Increased signal on top of that

- Pushes the peak out past the xenon background
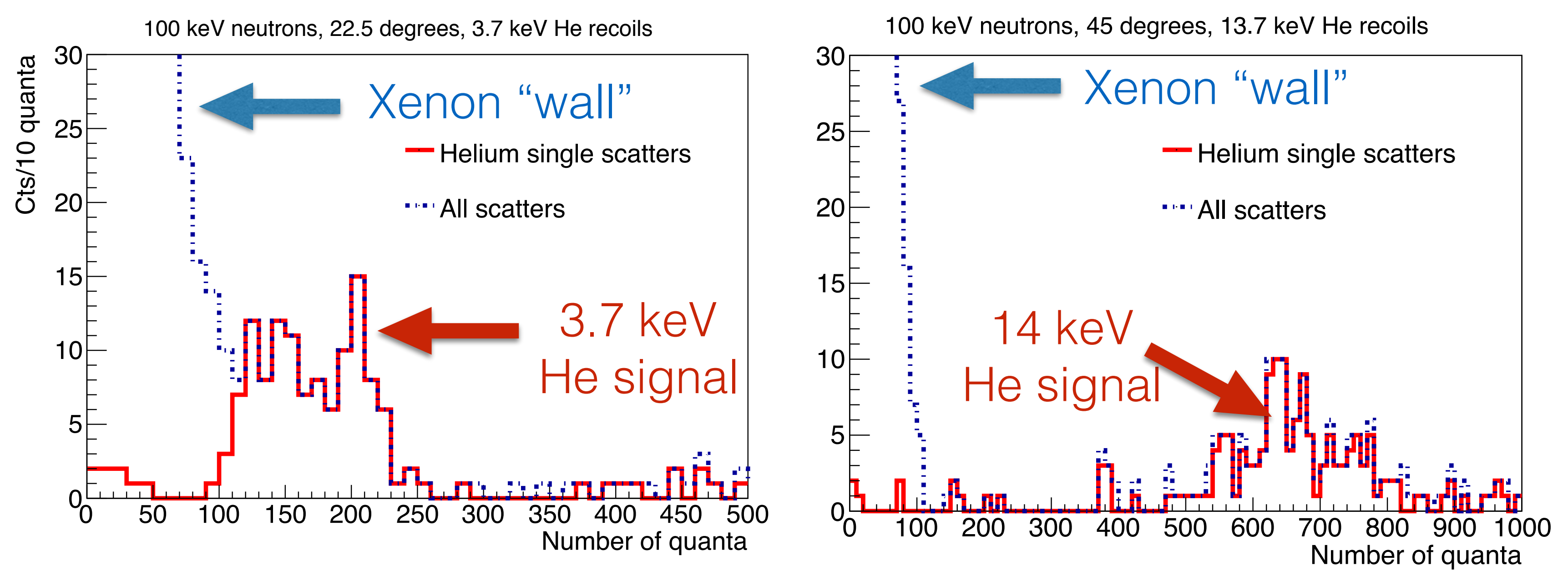

Measures yield and S1/S2 response v. energy! 